\title{
On Nonlinear Differential Equations, the Maximum Operation, and Monotone Convergence
}

\author{
ROBERT KALABA
}

Communicated by R. BeLlmaN

\section{Table of Contents}

Summary . . . . . . . . . . . . . . . . . 519

I. Introduction . . . . . . . . . . . . . . . . 521

1. Introduction. The Riccati Equation . . . . . . . . . . . . . 521

2. Convex and Concave Functions . . . . . . . . . . . . . . . 528

II. Ordinary Differential Equations . . . . . . . . . . 529

3. The Equation $u^{\prime}=f(u, x), u(0)=c \ldots \ldots \ldots \ldots$

4. The Equation $u^{\prime \prime}=f(u, x), u(0)=u(b)=0 \ldots \ldots$. . . . 538

5. The Equation $u^{\prime \prime}=f\left(u^{\prime}, u, x\right), u(0)=u(b)=0 \ldots \ldots 54$

III. Partial Differential Equations . . . . . . . . . . 550

6. The Equation $u_{x x}+u_{y y}=f(u, x, y) \ldots \ldots \ldots$

7. The Equation $u_{t}-u_{x x}=f(u, x, t) \ldots \ldots \ldots$

IV. Abstract Formulation and Discussion . . . . . . . . 560

8. The Equation $L[u]=f(u, x) \quad \ldots \ldots \ldots$

9. Discussion . . . . . . . . . . . . . . . . 565

Bibliography .................. . . 573

Summary. It is shown that the solutions of certain classes of nonlinear ordinary and partial differential equations may be represented in terms of the maximum operation applied to the solutions of associated linear equations. This, in effect, affords a new approach to the quasilinearization of nonlinear differential equations. This technique was first used by BeLLMAN, [10], who obtained, in particular, 
a representation for the solution of the initial value problem for the Riccati equation by its use.

The representation readily yields uniform lower bounds for solutions and, in the case of stochastic nonlinear differential equations, leads to representations for the distribution functions of the solutions. Lastly, but perhaps most important, a technique is provided for the construction of monotone sequences of functions which converge to the solutions of the nonlinear equation. In addition, the approximation scheme, which proves to be abstractly equivalent to Newton's method for the extraction of roots, is quadratically convergent and provides a method for the solution of nonlinear boundary value problems which is of interest from the computational viewpoint. Results of several numerical experiments carried out on a high speed digital computing machine are provided which tend to show that each additional iteration approximately doubles the number of correct digits.

The nonlinear equations considered are of the form

$$
L[u]=f(u, x),
$$

though various generalizations are indicated, where $x$ is an $n$-dimensional vector and $u$ is an unknown function to be determined on a domain $D$. The function $f(u, x)$ is considered to be a twice differentiable strictly convex (or concave) function of $u$ for all finite values of $u$ and all $x \varepsilon D$, and is continuous in $(u, x)$. The linear differential operator $L$ is to possess the positivity property

$$
L[z]-f^{\prime}(v) z \geqq 0 \Rightarrow z \geqq 0,
$$

which is to hold for all functions $v$ which are defined on $D$ and belong to an admissible set of functions $S$. The proof of the positivity property is not always straightforward; in some cases it corresponds to the non-negativity of Green's function on the domain $D$. Various initial and boundary values are considered.

The associated linear equation for the function $w=w(x)=w[x ; v]$ has the form

$$
L[w]-f^{\prime}(v) w=f(v)-v f^{\prime}(v),
$$

where $w$ is subject to the same boundary values as the function $u$. The representation of $u$ in the form

$$
u=\operatorname{Max}_{v \in S} w[x ; v],
$$

under appropriate restrictions, is demonstrated, which provides the central theme of the investigation.

The method is applied systematically to a variety of nonlinear equations including the Riccati equation

$$
\frac{d u}{d x}=u^{2}+g(x)
$$


and the equation

$$
\frac{d^{2} u}{d x^{2}}=f(u, x), \quad u(0)=u(b)=0 .
$$

Also considered are the nonlinear potential equation

$$
\Delta u=f(u)
$$

and the nonlinear heat equation

$$
u_{t}-u_{x x}=f(u) \text {. }
$$

In addition, when it is applied to the equation

$$
u_{t}+[f(u)]_{x}=0,
$$

we are able to rederive a representation formula for weak solutions of first order partial differential equations due to LAx.

Finally, generalizations to systems of first order nonlinear differential equations, nonlinear integral equations and variational problems are considered, and the role of the Min-Max operation in achieving quasilinearization is indicated.

\section{INTRODUCTION}

1. Introduction. The Riccati Equation. Nonlinear differential equations are playing an increasingly important role in both theory and applications $[18,22,43,44]$. In contrast, however, to the highly developed theory of linear differential equations, no well-rounded general theory of nonlinear differential equations is at hand. The primary effort has been directed toward the study of certain special classes of equations with particular emphasis upon those for which the nonlinear terms may be treated as mild perturbations about the linear ones. In one way or another, one usually seeks to linearize a given nonlinear problem.

We shall introduce a new linearization technique based upon the use of the maximum operation.

The intimate connection which exists between the theory of differential equations and variational problems has long been recognized. In particular, solutions of variational problems may be characterized as solutions of Euler differential equations, and by use of this connection the existence of solutions of differential equations may be established by the direct methods of the calculus of variations, making use, for example, of the Rayleigh-Ritz method. Furthermore, eigenfunctions and eigenvalues can be characterized by various variational principles (see, for example, Courant-Hilbert, [15], Pólya-Szegö, [38]).

Here we follow an entirely different path. We shall establish representation theorems for the solutions of various nonlinear ordinary and partial differential equations in terms of the maximum operation applied to the solutions of associated linear equations, and shall indicate some of the uses to which the 
representation may be put. The Poincaré-Perron method of superfunctions, [34], is related to our approach in that solutions of differential equations are represented as greatest lower bounds of superfunctions. We shall use our approach to provide a new practical method for numerically calculating solutions of nonlinear equations, and for other purposes.

In various branches of analysis, physics and engineering, equations of the form

$$
L[u]=f
$$

occur, where $L$ is a linear differential operator possessing a certain positivity property to be specified later (see Part IV), $f$ is a sufficiently smooth convex or concave function of $u$ and its derivatives (for all values of the independent variables throughout the domain of definition, should the independent variables occur explicitly), and $u$ is the unknown state variable. As examples we mention the Riccati equation,

$$
u^{\prime}=u^{2}+b(x) u+c(x),
$$

fundamental in the study of linear second order differential equations, and the equation

$$
u_{x x}+u_{y y}=e^{u}
$$

which occurs in studies of electrohydrodynamics, [24], and the physics of semiconductors, and which is of historic interest due to its treatment by PICARD in his classic memoir on the method of successive approximations, [35].

We shall study various ordinary and partial differential equations having the above-mentioned form, with the solutions subject to a variety of boundary conditions. In particular, we shall obtain a representation for solutions of these nonlinear equations in terms of the maximum or minimum operator applied to solutions of associated linear equations, a technique introduced by BELLMAN, $[9,10]$, in 1955 . This representation is then used to obtain the following types of results for solutions of nonlinear differential equations: uniform upper or lower bounds on solutions, nonlinear integral equations for the solutions, and representations for distribution functions of solutions of nonlinear stochastic differential equations.

Lastly, and perhaps most important, we are led to the construction of monotone sequences of functions which converge to solutions of the nonlinear equations, enabling us to provide certain existence theorems. These sequences are not only monotone, but also quadratically convergent, in HARTREE's terminology, so that they converge very rapidly, the number of correct digits in the approximation asymptotically doubling with each iteration. We refer to them as "Newtonian sequences", by analogy with the sequence of approximations to the root of a function provided by Newton's method (see Kantorovič, [21]). We illustrate this by giving the results of a number of numerical experiments carried out on the Johnniac, a high speed digital electronic computing machine 
at The RAND Corporation, of Princeton-type. In particular, we call attention to the results in Section 4 concerning the solution of a two-point boundary value problem for the equation $u^{\prime \prime}=e^{u}$ in which two iterations, involving sixteen seconds of machine calculation, yielded six figure accuracy.

A concrete example in the form of the Riccati equation provides an effective vehicle for the exposition of the methodology which we shall systematically exploit. We consider the equation

$$
u^{\prime}=u^{2}+g(x), \quad u(0)=c, \quad 0 \leqq x \leqq b,
$$

where $g(x)$ is continuous on the interval $[0, b]$ and $b$ is sufficiently small so as to ensure the existence of the solution. Since $(u-v)^{2} \geqq 0$, for any choice of the function $v$, we may write equation (1.2) in the form

$$
u^{\prime}=\operatorname{Max}\left\{2 u v-v^{2}+g(x)\right\}, \quad u(0)=c,
$$

where the maximization is over all functions $v$ defined on the interval $[0, b]$. The maximum is actually attained for $v=u$. The function $w=w(x)=w[x ; v]$ is then introduced as the solution of the associated linear equation

$$
w^{\prime}=2 v w-v^{2}+g(x), \quad w(0)=c,
$$

which we obtain by dropping the "Max" in equation (1.3). We wish to show first that $u$ may be represented in the form

$$
u=\operatorname{Max}_{v} w[x ; v],
$$

where the class of admissible functions $v$ contains $u$ and all other functions defined on the interval $[0, b]$ for which the integrals in equation (1.9) below exist.

First observe that equation (1.3) may be written

$$
u^{\prime}=2 u v-v^{2}+p+g(x), \quad u(0)=c,
$$

where $p$ is a non-negative function on the interval $[0, b]$ for any choice of the function $v$. It follows from equations (1.4) and (1.6) that the difference $u-w$ satisfies the equation

$$
(u-w)^{\prime}=2 v(u-w)+p, \quad u(0)-w(0)=0,
$$

for which the solution is

$$
u-w=\int_{0}^{x} p(s) \exp \left\{2 \int_{s}^{x} v(t) d t\right\} d s .
$$

The non-negativity of this integral establishes that $u \geqq w$. In addition, we see that if $v=u$ in equation (1.4), then the unique solution is $w=u$. Our proof of the representation in equation (1.5) is now complete. We may solve equation (1.4) for $w$ explicitly to obtain the following representation for the solution of the Riccati equation (1.2) in terms of integrals and a maximization: 


$$
\begin{aligned}
u(x)=\operatorname{Max}_{v} & \left(c \exp \left\{2 \int_{0}^{x} v(s) d s\right\}\right. \\
& \left.+\int_{0}^{x}\left[g(s)-v^{2}(s)\right] \exp \left\{2 \int_{s}^{x} v(t) d t\right\} d s\right), \quad 0 \leqq x \leqq b .
\end{aligned}
$$

It follows that a uniform lower bound for the function $u(x)$ on the interval $[0, b]$ may be obtained by evaluating the expression in brackets for any particular admissible choice of the function $v$. In addition, since the maximum occurs for $v=u, u$ satisfies the integral equation

$$
\begin{aligned}
u=c \exp \left\{2 \int_{0}^{x} u(s) d s\right\} \\
+\int_{0}^{x}\left[g(s)-u^{\prime}(s)\right] \exp \left\{2 \int_{s}^{x} u(t) d t\right\} d s, \quad 0 \leqq x \leqq b .
\end{aligned}
$$

It is a simple matter to verify that the solution of this integral equation satisfies equation (1.2).

A clue to the application of the foregoing techniques to nonlinear stochastic differential equations is provided by the fact that if $g(x), u(x), v(x)$, and $w(x)=$ $w[x ; v]$ are random functions,

$$
\operatorname{Prob}\{u(x) \geqq y\} \geqq \operatorname{Prob}\{w(x) \geqq y\}, \quad-\infty<y<+\infty,
$$

since $u(x) \geqq w[x ; v]$, whatever the random function $v$. This leads to the representation

$$
\operatorname{Prob}\{u(x) \geqq y\}=\underset{v}{\operatorname{Max}} \operatorname{Prob}\{w[x ; v] \geqq y\},
$$

which is discussed in Section 3, where an application to wave propagation through randomly inhomogeneous media is suggested, [11].

We now turn to the question of constructing monotone sequences of functions which converge to $u$; that is, we seek convergent minimizing sequences for the expression in brackets in equation (1.9). From the heuristic point of view it is convenient at this point to adopt some terminology from the theory of multistage decision processes of continuous type. Recalling equation (1.4), we shall refer to $v(x)$ as a policy-function and $w(x)$ as the corresponding return-function, with $u(x)$ in equation (1.3) being the return from an optimal policy, [6]. Note also that $u$ itself is the optimal policy-function.

Choose an arbitrary policy-function $v_{0}(x)$ and determine the corresponding return-function from equation (1.4). Call this return-function $w=u_{0}(x)$. Then use this return-function to determine an improved policy-function $v_{1}(x)$ as the function $v$ which maximizes $2 u_{0} v-v^{2}+g(x)$. This maximizing function is $v=v_{1}=u_{0}$. Compute the return-function $u_{1}$, using the policy-function $u_{0}$, as the solution of the equation

$$
u_{1}^{\prime}=2 u_{0} u_{1}-u_{0}^{2}+g(x), \quad u_{1}(0)=c .
$$


Then, using the return-function $u_{1}$, determine an improved policy-function $v_{2}(x)$ as the function $v$ which maximizes $2 u_{1} v-v^{2}+g(x)$. This maximizing function is $v=v_{2}=u_{1}$. Continuing in this way, we are led to consider the sequence of function $\left\{u_{n}\right\}$ defined by the equations

$$
\begin{array}{rlrl}
u_{0}^{\prime} & =2 v_{0} u_{0}-v_{0}^{2}+g(x), & u(0) & =c, \\
u_{n+1}^{\prime} & =2 u_{n} u_{n+1}-u_{n}^{2}+g(x), \quad u_{n+1}(0) & =c, \quad n=0,1,2, \cdots .
\end{array}
$$

BELLMAN has appropriately named this procedure "approximation in policy space" since a sequence of steadily improving policy-functions is obtained, the policies converging to $u$, which is itself the optimal policy, [7]. Let us note in passing that the method of steepest descent can be considered to be a particular type of approximation in policy space.

This sequence is uniformly bounded on an interval $[0, b]$ and converges uniformly and monotonically to the solution of equation (1.2), as will be shown in Part II. These properties can be used to establish an existence and uniqueness proof for solutions of equation (1.2). The Newtonian sequence $\left\{u_{n}\right\}$ is of interest in itself, providing, as it does, a modification of the more customary Picard successive approximation scheme,

$$
y_{n+1}^{\prime}=y_{n}^{2}+g(x), \quad y_{n+1}(0)=c, \quad n=0,1,2, \cdots,
$$

with significant computational advantages. If we introduce the metric

$$
\operatorname{Max}_{0 \leqq x \leqq b}\left|u-u_{n}\right|=\left\|u-u_{n}\right\|,
$$

then we shall show that

$$
\left\|u-u_{n}\right\| \leqq k\left\|u-u_{n-1}\right\|^{2}
$$

where $k$ is a constant independent of $n$, which implies the quadratic convergence of the sequence $\left\{u_{n}\right\}$. This shows that the number of correct digits in the approximation of $u_{n}$ to $u$ is asymptotically doubled with each iteration. As we remarked earlier, the method appears promising from the point of view of numerical computation, especially for two-point boundary value problems. The relatively slow convergence of the sequence $\left\{y_{n}\right\}$, obtained from conventional iterative techniques, militates against its use as a practical computational tool. The ideas discussed above, however, would seem to open the door to a greater use of the method of successive approximations in the computational solution of differential equations.

Let us briefly sketch one last application, the representation of the solution of the linear homogeneous equation of second order

$$
y^{\prime \prime}+g(x) y=0, \quad y(0)=y_{0} \neq 0, \quad y^{\prime}(0)=y_{1},
$$

through use of the minimum operation. This equation is of fundamental importance in the study of wave propagation through inhomogeneous media. In particular, this will illustrate that by use of a suitable transformation it may be 
possible to transform a given equation into a form appropriate for the application of our methodology.

We apply the well known substitution

$$
-\frac{y^{\prime}}{y}=u
$$

to bring equation (1.18) into the form of the Riccati equation

$$
u^{\prime}=u^{2}+g(x), \quad u(0)=-\frac{y_{1}}{y_{0}}=c .
$$

Physically, this transformation corresponds to introducing an impedance in place of the wave function. The representation that we have given for the solution of Riccati's equation leads to a new type of minimum principle-a principle of minimum impedance in contradistinction to the usual energy principles. From the previous discussion we know that the function $u$ may be represented on a sufficiently small interval $[0, b]$ in the form

$$
u=\operatorname{Max}_{v} w[x ; v]
$$

where $w=w[x ; v]$ is the solution of the equation

$$
w^{\prime}=2 v w-v^{2}+g(x), \quad w(0)=c .
$$

Observing that the inverse of the transformation (1.19) is given by

$$
y=y_{0} \exp \left\{-\int_{0}^{x} u(t) d t\right\}
$$

we find that

$$
\frac{y}{y_{0}}=\exp \left\{-\int_{0}^{x}[\underset{v}{\operatorname{Max}} w[s ; v] d s]\right\}, \quad 0 \leqq x \leqq b,
$$

from which it follows that

$$
\frac{y}{y_{0}}=\operatorname{Min}_{v} \exp \left\{-\int_{0}^{x} w[s ; v] d s\right\} .
$$

By solving equation (1.22) for $w$ we find that the solution of equation (1.18) may be represented in the form

$$
\begin{aligned}
\frac{y}{y_{0}}= & \operatorname{Min}_{v} \exp \left\{-\int_{0}^{x}\left[c \exp \left\{2 \int_{0}^{s} v(t) d t\right\}\right.\right. \\
& \left.\left.+\int_{0}^{s}\left(g(t)-v^{2}(t)\right) \exp \left\{2 \int_{t}^{s} v\left(t_{1}\right) d t_{1}\right\} d t\right] d s\right\}, \quad 0 \leqq x \leqq b .
\end{aligned}
$$

It is clear that this consideration affords a new approach to the approximation of solutions of equation (1.18), based, for example, on the known convergent 
minimizing sequence $\left\{u_{n}\right\}$ defined by equation (1.14). (Cf. the standard Liouville (W.K.B.) technique, that of Schelkunofr, [40], and that of BAILEY, [4].)

Observe that we tacitly made use above of the existence and uniqueness of solutions of equations (1.2) and (1.4) on the interval $[0, b]$. In addition, the verification of the positivity property expressed by equations (1.7) and (1.8) was quite simple. In applications to other equations, though frequently existence and uniqueness theorems are available, the establishing of the positivity property constitutes a major obstacle. In some instances it corresponds to showing that Green's or related functions do not change sign in the domain under consideration (cf. Aronszajn \& Smith, [1, 2]).

Extensions of the discussion above will be given in Part II, which is devoted to nonlinear ordinary differential equations. The equations to be considered there are of the forms

$$
\begin{aligned}
u^{\prime}(x) & =f(u, x), & & u(0)=c, \\
u^{\prime \prime}(x) & =f(u, x), & & u(0)=u(b)=0, \\
u^{\prime \prime}(x) & =f\left(u^{\prime}, u, x\right), & & u(0)=u(b)=0 .
\end{aligned}
$$

It is assumed that the function $f$ is strictly convex or concave as a function of $u$ and $u^{\prime}$ (if present) for all finite $u$ and $u^{\prime}$ and for all values of $x$ in the interval $[0, b]$, and that it has bounded second partial deviatives with respect to $u$ and $u^{\prime}$ for all finite values of the arguments. This last restriction can be relaxed to requiring the boundedness of the second derivatives in an interval $m_{1} \leqq u$, $u^{\prime} \leqq m_{2}$, provided that we have $a$ priori knowledge that the solution satisfies this inequality.

The proof of the positivity property for the operator $d^{2} / d x^{2}+q(x)$ in a sufficiently small interval, zero boundary conditions being imposed, is given as Lemma 4.1 of Section 4 in the form of a statement concerning the positivity of Green's function. A transformation is used to reduce equation (1.29), under certain circumstances, to equation (1.28), which is discussed in Section 5 . Extensions are indicated in the Discussion of Part IV.

Part III is devoted to a consideration, along similar lines, of applications to nonlinear partial differential equations. In Section 6 we prove various representation and approximation theorems concerning the nonlinear elliptic equation

$$
u_{x x}+u_{y y}=f(u, x, y) .
$$

In Section 7 we do the same for the nonlinear parabolic equation

$$
u_{t}-u_{x x}=f(u, x, t) \text {. }
$$

The proofs of the positivity lemmas for the corresponding linear equations are of interest per se.

Having considered applications of the techniques to a wide variety of nonlinear ordinary and partial differential equations, in Part IV we provide an abstract formulation which serves the purpose of highlighting the properties 
required of the operator $L$ and the function $f$ for the technique-linearization of a problem through maximization-to be applicable. In the discussion of Section 9 that follows additional applications are briefly sketched. These include applications to the calculus of variations, nonlinear integral equations, quasilinear first order partial differential equations, and systems of ordinary differential equations. Use of the Min-Max operation for achieving linearization is sketched. Lastly, some additional positivity results are mentioned.

Acknowledgement. The author takes this opportunity to express very gratefully his sincerest thanks to many individuals at New York University and elsewhere for their invaluable aid. Professor P. D. LAx provided much helpful advice and, in particular, indicated the applications to the theory of weak solutions of nonlinear equations.

Several papers by Dr. Rrchard Bellman on functional equations provided the stimulus for this work. Furthermore, his personal unflagging interest and guidance have been most decisive throughout the entire investigation.

Much invaluable insight was gained through conversations with Professors A. Douglis, W. Prager, A. J. F. Siegert, and E. Reich.

Dr. M. L. Juncosa made many useful suggestions concerning the numerical aspects of the work. The computations were very capably carried through by M. B. Shapiro, L. Slade, and P. H. Haley.

2. Convex and Concave Functions. In the extensions of the treatment of the Riccati equation to various other nonlinear ordinary and partial differential equations of the form $L[u]=f(u, x)$, where $L$ is a linear operator, we shall assume that $f$ is a strictly convex function of $u$ for all $x$ in a domain $D$ and for all $u$ that enter into consideration. In addition, we shall assume that $f$ has a bounded second partial derivative with respect to $u$ (in connection with the quadratic convergence). In general, we shall not specifically indicate the dependence of $f$ on $x$ and shall indicate partial derivatives with respect to $u$ by primes.

The property of the strictly convex function $f$, which is of paramount importance in the following, we state as

Lemma 2.1 If $f(u)$ is strictly convex and twice differentiable in any finite $u$ interval, then $f(u)$ may be represented in the form

$$
f(u)=\operatorname{Max}_{v}\left[f(v)+(u-v) f^{\prime}(v)\right],
$$

where the maximization is over all $v$; the maximum is actually attained for $v=u$.

The geometric meaning of this is that for a strictly convex curve the point at which an arbitrary tangent cuts a given parallel to the ordinate axis is not above the point at which the curve cuts the given parallel.

The proof is simple. By use of the mean value theorem we may write $f(u)$ in the form

$$
f(u)=f(v)+f^{\prime}(v)(u-v)+\frac{1}{2} f^{\prime \prime}\left(v_{1}\right)(u-v)^{2},
$$


where $v_{1}$ lies between $v$ and $u$. The strict convexity assumption implies that

$$
f^{\prime \prime}(u)>0
$$

so that

$$
f(u)-\left[f(v)+f^{\prime}(v)(u-v)\right] \geqq 0,
$$

with the equality holding for $v=u$. This is precisely the content of the lemma.

For strictly concave functions a similar representation in valid:

$$
f(u)=\operatorname{Min}_{v}\left[f(v)+(u-v) f^{\prime}(v)\right]
$$

We also require a similar representation for strictly convex twice differentiable functions of $n$ variables $f(v)=f\left(v_{1}, v_{2}, \cdots, v_{n}\right)$. By definition of strict convexity, the Hessian matrix $\left[\partial^{2} f / \partial v_{i} \partial v_{i}\right]$ is positive-definite, so that

$$
f(u) \geqq f(v)+\sum_{i=1}^{n}\left(u_{i}-v_{i}\right) \frac{\partial f}{\partial v_{i}},
$$

with equality holding only for $v=u$. This leads to the representation

$$
f(u)=\operatorname{Max}_{v}\left[f(v)+\sum_{i=1}^{n}\left(u_{i}-v_{i}\right) \frac{\partial f}{\partial v_{i}}\right]
$$

where the maximization is over all $v$.

A similar representation holds for strictly concave functions of $v=\left(v_{1}, v_{2}\right.$, $\left.\cdots, v_{n}\right)$. In general, though, we shall consider only convex functions in subsequent sections. We use these representations to aid in the linearization of various nonlinear problems, as will become evident.

\section{Ordinary Differential Equations}

Our first application of the technique sketched above is to several nonlinear ordinary differential equations of the first and second orders. As a direct generalization of the Riccati equation (1.2) we consider the equation

$$
u^{\prime}=f(u, x), \quad u(0)=c, \quad 0 \leqq x \leqq b,
$$

where the function $f$ is strictly convex as a function of $u$ for all values of $u$ and $x$ which come into consideration. In addition, $f$ is assumed continuous as a function of $u$ and $x$, and it is assumed to have a bounded second partial derivative with respect to $u$. The independent variable $x$ is limited to a sufficiently small interval $[0, b]$ to insure the existence and uniqueness of a solution of the equation. We establish a representation theorem for the solution, employing the maximum operation, and then use this representation to establish lower bounds on the solution and a nonlinear integral equation satisfied by $u$, and to construct a monotone sequence of junctions $\left\{u_{n}\right\}$ converging to $u$. Finally, a representation for the distribution function of the solution of the nonlinear 
stochastic differential equation

$$
u^{\prime}=u^{2}+r(t), \quad u(0)=c,
$$

where $r(t)$ is a random function of $t$, is provided.

Following this we indicate the treatment of a two-point boundary value problem for a nonlinear differential equation of second order,

$$
u^{\prime \prime}=f(u, x), \quad u(0)=u(b)=0,
$$

where $f$ is subject to the usual convexity and regularity conditions. Interest here centers on proving the requisite positivity property; namely, if the function $z$ satisfies the differential inequality

$$
z^{\prime \prime}-f_{v}(v, x) z \geqq 0,
$$

and the boundary conditions

$$
z(0)=z(b)=0
$$

then $z(x)$ is non-positive for $0 \leqq x \leqq b$, provided $b$ is sufficiently small. This lemma is used to establish the representation theorem, from which a number of results follow. In particular, a rapidly convergent monotone sequence of functions is constructed which is promising as a practical means for the numerical solution of certain boundary value problems, which is illustrated by the results of a numerical experiment.

Following this we indicate the treatment of the equation

$$
u^{\prime \prime}=f\left(u^{\prime}, u, x\right), \quad u(0)=u(b)=0,
$$

under the principal assumption that $f$ is strictly convex as a function of $u^{\prime}$ and $u$. Essentially we reduce this to the previously considered case.

3. The Equation $u^{\prime}=f(u, x), u(0)=c$. Let us consider the equation

$$
u^{\prime}=f(u, x), \quad u(0)=c, \quad 0 \leqq x \leqq b,
$$

where $f$ is assumed to be continuous in $u$ and $x$, to have a bounded second partial derivative with respect to $u$ for all $u$ and $x$ that enter into the discussion, and to be strictly convex in $u$. We shall first make use of the well known result that a unique continuous function $u(x)$ exists which satisfies equation (3.1), provided that $b$ is sufficiently small, though later we shall provide a proof of this.

Representation Theorem. We shall now establish a representation theorem for $u$ which generalizes that provided for the Riccati equation discussed in the introduction. In accord with Section 2 we may represent the function $f(u, x)$ in the form

$$
f(u, x)=\operatorname{Max}_{v}\left\{f(v, x)+(u-v) f_{v}(v, x)\right\},
$$


where the maximization is over all functions defined on $[0, b]$. In the interest of brevity, let us no longer indicate the explicit dependence of $f$ on $x$ and write equation (3.2) as

$$
f(u)=\operatorname{Max}_{v}\left\{f(v)+(u-v) f^{\prime}(v)\right\}
$$

where the prime indicates partial differentiation with respect to $v$. Then equation (3.1) may be rewritten

$$
u^{\prime}=\operatorname{Max}_{v}\left\{f(v)+(u-v) f^{\prime}(v)\right\}, \quad u(0)=c .
$$

The maximum is actually assumed for $v=u$.

We now introduce the function $w=w(x)=w[x ; v]$ defined to be the solution of the associated linear equation which we obtain by dropping the "Max" in equation (3.4):

$$
w^{\prime}=f(v)+(w-v) f^{\prime}(v), \quad w(0)=c,
$$

or

$$
w^{\prime}-f^{\prime}(v) w=f(v)-v f^{\prime}(v), \quad w(0)=c .
$$

The solution is

$$
\begin{aligned}
& w[x ; v]=c \exp \left\{\int_{0}^{x} f^{\prime}(v(s)) d s\right\} \\
& \quad+\int_{0}^{x}\left[f(v(s))-v(s) f^{\prime}(v(s))\right] \exp \left\{\int_{s}^{x} f^{\prime}(v(t)) d t\right\} d s .
\end{aligned}
$$

Each function $v(x)$ determines a unique solution $w=w[x ; v]$, the only restriction on the admissibility of $v$ being the integrability of the functions $f(v(x)), f^{\prime}(v(x))$, and $v(x) f^{\prime}(v(x))$ on the interval $[0, b]$, which we shall henceforth assume.

Let us now demonstrate

Theorem 3.1. The solution $u$ of equation (3.1) is given by

$$
\begin{aligned}
u(x) & =\operatorname{Max}_{v} w[x ; v]=\operatorname{Max}_{v}\left(c \exp \left\{\int_{0}^{x} f^{\prime}(v(s)) d s\right\}\right. \\
& \left.+\int_{0}^{x}\left[f(v(s))-v(s) f^{\prime}(v(s))\right] \exp \left\{\int_{s}^{x} f^{\prime}(v(t)) d t\right\} d s\right), \quad 0 \leqq x \leqq b,
\end{aligned}
$$

where the maximization is over all admissible functions $v(x)$ defined on the interval $0 \leqq x \leqq b$. The maximum is attained for $v=u$ itself.

This is a straightforward generalization of the representation given earlier for the solution of the Riccati equation.

Proof. For any admissible choice of the function $v(x)$ equation (3.4) may be rewritten

$$
u^{\prime}=f(v)+(u-v) f^{\prime}(v)+p
$$


where $p$ is a non-negative function for any particular choice of the function $v$. If we introduce the difference function

$$
z=u-w
$$

we see from equations (3.5) and (3.9) that $z$ satisfies the equation

$$
z^{\prime}=f^{\prime}(v) z+p, \quad z(0)=0 .
$$

Since the solution of this first order linear equation is given explicitly by the integral

$$
z=\int_{0}^{x} \exp \left\{\int_{s}^{x} f^{\prime}(v(t)) d t\right\} p(s) d s
$$

which is clearly non-negative, it is seen that

$$
z=u-w \geqq 0,
$$

or

$$
u \geqq w[x ; v],
$$

for all admissible $v$ on the interval $[0, b]$.

In remains to show that the function $v(x)$ can be chosen to yield equality in equation (3.12). If we choose $v$ to be equal to $u$ itself in equation (3.5), the result is

$$
w^{\prime}-f^{\prime}(u) w=f(u)-u f^{\prime}(u), \quad w[0 ; u]=c,
$$

the unique solution of which is seen to be

$$
w=u \text {. }
$$

The relations (3.14) and (3.16) taken together establish the representation in equation (3.8).

Corollaries. It is evident that the function $w$ corresponding to any admissible choice of the function $v$, e.g. $v=v_{1}$, provides a uniform lower bound for the solution $u$ on the interval $[0, b]$ :

$$
u \geqq w\left[x ; v_{1}\right] .
$$

Indeed, as will be shown below, a monotone increasing sequence of functions converging to $u$ can be constructed.

In addition, since the maximizing function $v$ is $u$ itself, $u$ satisfies the nonlinear integral equation

$$
\begin{aligned}
& u(x)=c \exp \left\{\int_{0}^{x} f^{\prime}(u(s)) d s\right\} \\
& \quad+\int_{0}^{x}\left[f(u(s))-u(s) f^{\prime}(u(s))\right] \exp \left\{\int_{s}^{x} f^{\prime}(u(t)) d t\right\} d s
\end{aligned}
$$

which can be verified directly. 
Stochastic Differential Equations. Let us now see how the technique used above, involving the representation of solutions of non-linear differential equations through use of the maximum operator applied to the solutions of associated linear equations, can be used to obtain a representation of the distribution function for the solution of a nonlinear stochastic differential equation, [11]. We focus our attention on the special equation of particular importance.

$$
u^{\prime}=u^{2}+r(t), \quad u(0)=c,
$$

where $r(t)$ is a random function with a given distribution function. The stochastic Riccati equation (3.19) is associated with the linear equation

$$
\frac{d^{2} z}{d x^{2}}+r(x) z=0
$$

which plays a role in the study of wave propagation through randomly inhomogeneous media.

As we have seen, we may represent the solution of equation (3.19) in the form

$$
u=\operatorname{Max}_{v} w[t ; v],
$$

where $w=w[t ; v]$ is the solution of the associated linear equation

$$
w^{\prime}-2 v w=r(t)-v^{2}, \quad w[0 ; v]=c .
$$

If we now let

$$
\begin{aligned}
W(t, y ; v) & =\operatorname{Prob}\{w[t ; v] \geqq y\}, \\
U(t, y) & =\operatorname{Prob}\{u(t) \geqq y\}, \quad-\infty<y<+\infty,
\end{aligned}
$$

it is clear from the representation (3.21) that

$$
U(t, y) \geqq W(t, y ; v),
$$

for all random functions $v(t)$. In addition, we have equality when $v=u$. Thus we are led to

Theorem 3.2. If $u(t)$ is the random function solution of the stochastic differential equation (3.19), assumed to exist for $0 \leqq t \leqq T$, then, for $0 \leqq t \leqq T$ and for $-\infty<y<+\infty$, the representation

$$
\operatorname{Prob}\{u(t) \geqq y\}=\operatorname{Max}_{v} \operatorname{Prob}\{w[t ; v] \geqq y\}
$$

holds, where the maximization is over all random functions $v(t)$ defined on the interval $0 \leqq t \leqq T$.

It is clear that the representation (3.25) leads to lower bounds for the probability of the inequality $u(t) \geqq y$. The method is readily extended to other nonlinear stochastic differentials and functional equations, and to the linear stochastic equation (3.20). 
Newtonian Approximation. Now let us show how to construct sequences of functions $\left\{u_{n}(x)\right\}$ which converge monotonically to $u(x)$, the solution of equation (3.1) on the interval $0 \leqq x \leqq b$. The sequence is referred to as "Newtonian" in view of its close conceptual connection with Newton's method for approximating a root of a function. With Newton's method it shares the properties of monotonicity and quadratic convergence.

Returning to the equation (3.5), select an initial function $v_{0}(x)$ and determine the function $u_{0}(x)$ as the solution of the equation

$$
u_{0}^{\prime}=f\left(v_{0}\right)+\left(u_{0}-v_{0}\right) f^{\prime}\left(v_{0}\right), \quad u_{0}(0)=c .
$$

Next, keeping the representation of equation (3.8) in mind and the equation (3.4), determine the function $v_{1}(x)$ as the function which maximizes the expression $f(v)+\left(u_{0}-v\right) f^{\prime}(v)$. In view of the assumed convexity of $f(v)$, this maximizing function is $v=v_{1}=u_{0}$. Determine the corresponding function $u_{1}$ as the solution of

$$
u_{1}^{\prime}=f\left(u_{0}\right)+\left(u_{1}-u_{0}\right) f^{\prime}\left(u_{0}\right), \quad u_{1}(0)=c .
$$

Next find $v_{2}$, the function which maximizes the expression $f(v)+\left(u_{1}-v\right) f^{\prime}(v)$. This function is $v_{2}=u_{1}$. Then the function $u_{2}$ is determined as the solution of

$$
u_{2}^{\prime}=f\left(u_{1}\right)+\left(u_{2}-u_{1}\right) f^{\prime}\left(u_{1}\right), \quad u_{2}(0)=c .
$$

Continuing in this way we are led to the sequence $\left\{u_{n}(x)\right\}$ determined by the recurrence relations

$$
\begin{aligned}
u_{0}^{\prime} & =f\left(v_{0}\right)+\left(u_{0}-v_{0}\right) f^{\prime}\left(v_{0}\right), & u_{0}(0) & =c, \\
u_{n+1}^{\prime} & =f\left(u_{n}\right)+\left(u_{n+1}-u_{n}\right) f^{\prime}\left(u_{n}\right), & u_{n+1}(0) & =c, \quad n=0,1,2, \cdots .
\end{aligned}
$$

From its mode of construction we expect the sequence $\left\{u_{n}\right\}$ to converge monotonically to $u(x)$. In addition, we observe that $f\left(u_{n+1}\right)$ is approximated by $f\left(u_{n}\right)+\left(u_{n+1}-u_{n}\right) f^{\prime}\left(u_{n}\right)$-allied to replacing a curve in the neighborhood of a point with its tangent, the essential feature of Newton's method-so that we may expect rapid convergence. Let us now demonstrate these conjectures.

We first establish, using classical techniques, the existence of a common interval $[0, b]$ on which the sequence $\left\{u_{n}\right\}$ is uniformly bounded. In particular, we show that

$$
\left\|u_{n}(x)-c\right\| \leqq 1, \quad 0 \leqq x \leqq b, \quad n=0,1,2, \cdots,
$$

where $b$ is sufficiently small and the norm is defined by

$$
\left\|u_{n}(x)-c\right\|=\operatorname{Max}_{0 \leqq x \leqq b}\left|u_{n}(x)-c\right| .
$$

Under our assumption concerning the boundedness of $f(v)$ and its first two derivatives for finite $v$, we may write

$$
\operatorname{Max}\left\{|f(v)|,\left|f^{\prime}(v)\right|, \frac{1}{2}\left|f^{\prime \prime}(v)\right|\right\} \leqq m<\infty
$$


for

$$
|v-c| \leqq 1
$$

Let $v_{0}(x)$ be chosen so that

$$
\left\|v_{0}(x)-c\right\| \leqq 1
$$

Then by integrating both sides of the first relation in equation (3.29) we find

$$
u_{0}(x)-c=\int_{0}^{x}\left[f\left(v_{0}(s)\right)+\left(u_{0}(s)-v_{0}(s)\right) f^{\prime}\left(v_{0}(s)\right)\right] d s, \quad 0 \leqq x .
$$

From this it follows that

$$
\left\|u_{0}(x)-c\right\| \leqq x\left[m+m\left(\left\|u_{0}-c\right\|+1\right)\right]
$$

so that

$$
\left\|u_{0}(x)-c\right\| \leqq \frac{2 b m}{1-m b}
$$

Hence

$$
\left\|u_{0}(x)-c\right\| \leqq 1
$$

provided that

$$
0 \leqq x \leqq b<\frac{1}{3 m} .
$$

We now proceed inductively. Under the assumption that

$$
\left\|u_{n}-c\right\| \leqq 1, \quad 0 \leqq x \leqq b,
$$

for a particular $n$, we find, as before, that

$$
u_{n+1}-c=\int_{0}^{x}\left[f\left(u_{n}(s)\right)+\left(u_{n+1}(s)-u_{n}(s)\right) f^{\prime}\left(u_{n}(s)\right)\right] d s, \quad 0 \leqq x \leqq b,
$$

so that

$$
\left\|u_{n+1}-c\right\| \leqq \frac{2 x m}{1-x m}
$$

which will not exceed unity provided relation (3.39) holds. This completes the induction which may be started for $n=0$.

From equation (3.5) which defines $w$, equation (3.29) which defines the sequence $\left\{u_{n}\right\}$, and the representation in equation (3.8), it is evident that

$$
u(x) \geqq u_{n}(x), \quad n=0,1,2, \cdots, \quad 0 \leqq x \leqq b .
$$

Thus $u(x)$ itself provides an upper bound on the sequence $\left\{u_{n}(x)\right\}$ in some interval for which $u(x)$ exists; furthermore, as we shall see from the monotonicity of the sequence, $u_{0}(x)$ provides a lower bound. Thus, if the boundedness were all 
we wished to obtain, the above argument would be superfluous. Later, though, in establishing the existence of a solution of equation (3.1), we shall have need for the uniform boundedness of the sequence $\left\{u_{n}\right\}$ without assuming the existence of $u$.

Monotonicity. With the uniform boundedness of the sequence $\left\{u_{n}\right\}$ in hand, let us proceed to show that the sequence is monotone increasing on the interval $[0, b]$. Referring to equation (3.29) we note that

$$
\begin{aligned}
u_{n+1}^{\prime} & =f\left(u_{n}\right)+\left(u_{n+1}-u_{n}\right) f^{\prime}\left(u_{n}\right), \\
u_{n}^{\prime} & =f\left(u_{n-1}\right)+\left(u_{n}-u_{n-1}\right) f^{\prime}\left(u_{n-1}\right) .
\end{aligned}
$$

Using the fact that the maximum of the expression $f(v)+(u-v) f^{\prime}(v)$, for $f(v)$ convex, occurs when $v=u$, we obtain from the last equation the inequality

$$
u_{n}^{\prime} \leqq f\left(u_{n}\right)+\left(u_{n}-u_{n}\right) f^{\prime}\left(u_{n}\right), \quad n=0,1,2, \cdots .
$$

By subtracting this inequality from the first relation in equation (3.43) we find

$$
\begin{gathered}
\left(u_{n+1}-u_{n}\right)^{\prime} \geqq\left(u_{n+1}-u_{n}\right) f^{\prime}\left(u_{n}\right), \quad 0 \leqq x \leqq b, \quad n=0,1,2, \cdots, \\
u_{n+1}(0)-u_{n}(0)=0 .
\end{gathered}
$$

As we have already seen, in connection with the positivity property expressed in equations (3.11) and (3.13), this implies that

$$
u_{n+1}(x)-u_{n}(x) \geqq 0, \quad 0 \leqq x \leqq b, \quad n=0,1,2, \cdots .
$$

This completes the proof of the first part of

Theorem 3.3. The sequence of functions $\left\{u_{n}(x)\right\}$ given by equation (3.29) is monotone increasing on the interval $[0, b]$ and converges to $u(x)$, the solution of equation (3.1).

In view of its uniform boundedness and monotonicity, the sequence $\left\{u_{n}(x)\right\}$ converges to a limit function $U(x)$ on the interval $[0, b]$. To complete the proof of Theorem 3.3 we must show that $U(x)$ satisfies equation (3.1). The proof follows standard lines. Integrate both sides of equation (3.29) from 0 to $x$ and use Lebesgue's theorem on bounded convergence to obtain the integral relation

$$
U(x)-c=\int_{0}^{x} f(U(t)) d t, \quad 0 \leqq x \leqq b .
$$

It follows, in order, that $U(x)$ is integrable, absolutely continuous, and differentiable on the interval $[0, b]$, so that

$$
U^{\prime}(x)=f(U(x)), \quad U(0)=c .
$$

This completes the proof that $U$ satisfies equation (3.1). The proof of uniqueness is well known. 
Quadratic Convergence. A sequence of functions $\left\{u_{n}\right\}$ is said to be quadratically convergent if it converges to a limit function $u$ and if

$$
\left\|u_{n}-u\right\| \leqq k\left\|u_{n-1}-u\right\|^{2},
$$

where $k$ is independent of $n$. Let us demonstrate

Theorem 3.4. The sequence $\left\{u_{n}\right\}$ is quadratically convergent on the interval $[0, b]$.

Proof. From equations (3.1) and (3.43) we find that

$$
\begin{array}{r}
\left(u-u_{n}\right)^{\prime}=f(u)-f\left(u_{n-1}\right)-\left(u_{n}-u_{n-1}\right) f^{\prime}\left(u_{n-1}\right), \\
0 \leqq x \leqq b, \quad n=0,1,2, \cdots .
\end{array}
$$

This can be rewritten to yield

$$
\left(u-u_{n}\right)^{\prime}=f(u)-f\left(u_{n-1}\right)-\left(u-u_{n-1}\right)-f^{\prime}\left(u_{n-1}\right)-\left(u_{n}-u\right) f^{\prime}\left(u_{n-1}\right) .
$$

Integrating from 0 to $x$, we find, since $u(0)-u_{n}(0)=0$,

$$
\left(u-u_{n}\right)=\int_{0}^{x}\left[f(u)-f\left(u_{n-1}\right)-\left(u-u_{n-1}\right) f^{\prime}\left(u_{n-1}\right)-\left(u_{n}-u\right) f^{\prime}\left(u_{n-1}\right)\right] d s .
$$

Consequently, making use of the mean value theorem, we see

$$
u-u_{n}=\int_{0}^{x}\left[\frac{1}{2} f^{\prime \prime}\left(\bar{q}_{n}\right)\left(u-u_{n-1}\right)^{2}+\left(u-u_{n}\right) f^{\prime}\left(u_{n-1}\right)\right] d s,
$$

where $\bar{q}_{n}$ lies between $u_{n-1}$ and $u$ for $x$ on the interval $0 \leqq x \leqq b$. In view of the relation (3.32) we may write

$$
\left\|u-u_{n}\right\| \leqq b\left\{m\left\|u-u_{n-1}\right\|^{2}+m\left\|u-u_{n}\right\|\right\}
$$

which yields

$$
\left\|u-u_{n}\right\| \leqq \frac{b m}{1-b m}\left\|u-u_{n-1}\right\|^{2} .
$$

This is equation (3.49) with $k=b m(1-b m)^{-1}$. Since $b$ was chosen so that $b m<\frac{1}{3}$, this also establishes the uniform convergence of the sequence on the interval $[0, b]$, under the assumption that $u$ is a solution of equation (3.1). The results of a numerical computation, given below, illustrate the fact that the number of digits of $u_{n}(x)$ in agreement with those of $u(x)$ on the interval $[0, b]$ is approximately doubled with each iteration.

Next let us demonstrate that

$$
\left\|u_{n+1}-u_{n}\right\| \leqq k_{1}\left\|u_{n}-u_{n-1}\right\|^{2}
$$

from which we can show, ab initio, the existence of a solution of equation (3.1). From equation (3.43) we obtain

$$
\left(u_{n+1}-u_{n}\right)^{\prime}=\left[f\left(u_{n}\right)-f\left(u_{n-1}\right)-\left(u_{n}-u_{n-1}\right) f^{\prime}\left(u_{n-1}\right)\right]+\left(u_{n+1}-u_{n}\right) f^{\prime}\left(u_{n}\right) .
$$


Then by integrating and using the mean value theorem we find, as before,

$$
\left\|u_{n+1}-u_{n}\right\| \leqq \frac{b m}{1-b m}\left\|u_{n}-u_{n-1}\right\|^{2}, \quad b m<\frac{1}{3}, \quad n=0,1,2, \cdots .
$$

This establishes the uniform convergence of the sequence $\left\{u_{n}\right\}$ on the interval $0 \leqq x \leqq b$. Consequently, by integrating both sides of equation (3.29) and passing to the limit, we find that $V(x)$, the continuous limit function of the continuous functions $u_{n}(x)$, satisfies the equation

$$
V(x)-c=\int_{0}^{x} f(V(s)) d s .
$$

The right hand side of this equation being differentiable, we find $V$ satisfies the equation

$$
V^{\prime}(x)=f(V(x)), \quad V(0)=c,
$$

which completes the proof.

Lastly we note that the usual Picard successive approximation scheme yields only convergence of the first order. Let us now examine the results of a numerical experiment.

Numerical Example. In order to investigate the rate of convergence of the sequence $\left\{u_{n}\right\}$ to $u$ numerically, the equation

$$
u^{\prime}=1+u^{2}, \quad u(0)=0,
$$

for which the solution is $u=\tan x$, was considered. The functions $u_{n}(x)$ were determined recursively as the solutions of the recurrence relations

$$
\begin{array}{rlrl}
u_{0}^{\prime} & =2 v_{0} u_{0}+\left(1-v_{0}^{2}\right), & u_{0}(0) & =0, \\
u_{n+1}^{\prime} & =2 u_{n} u_{n+1}+\left(1-u_{n}^{2}\right), \quad u_{n+1}(0) & =0, \quad n=0,1,2, \cdots,
\end{array}
$$

using a Runge -Kutta integration procedure capable of yielding $u_{n}(x)$ to somewhat over six figures of accuracy. The interval considered was $0 \leqq x \leqq 0.99$, and two initial approximations, $v_{0}=0$ and $v_{0}=x+\frac{1}{3} x^{3}$, the first terms in the power series expansion of $\tan x$ about the origin, were used.

The results are displayed in Tables 1 and 2 in which the digits of $u_{n}(x)$ in agreement with those of $\tan x$ are in italics.

4. The Equation $u^{\prime \prime}=f(u, x), u(0)=u(b)=0$. We now turn to a consideration of the nonlinear equation of second order

$$
u^{\prime \prime}=f(u, x), \quad 0 \leqq x \leqq b,
$$

where $u$ is subject to the two point boundary condition

$$
u(0)=u(b)=0 .
$$

We shall assume initially that if $b$ is sufficiently small then a unique solution 
TABLE 1.

The convergence of $u_{n}(x)$ to $u(x)=\tan x, v_{0}(x)=0$.

\begin{tabular}{c|c|c|c|c|c|c}
\hline$x$ & $v_{0}(x)$ & $u_{0}(x)$ & $u_{1}(x)$ & $u_{2}(x)$ & $u_{3}(x)$ & $\tan x$ \\
\hline 0.0 & .000000 & .000000 & .000000 & .000000 & .000000 & .000000 \\
.1 & .000000 & .100000 & .100335 & .100335 & .100335 & .100335 \\
.2 & .000000 & .200000 & .202710 & .202710 & .202710 & .202710 \\
\hline .3 & .000000 & .300000 & .309332 & .309336 & .309336 & .309336 \\
.4 & .000000 & .400000 & .422761 & .422793 & .422793 & .422793 \\
.5 & .000000 & .500000 & .546142 & .546302 & .546302 & .546302 \\
.6 & .000000 & .600000 & .683512 & .684136 & .684136 & .684137 \\
.7 & .000000 & .700000 & .840227 & .842287 & .842288 & .842288 \\
\hline .8 & .000000 & .800000 & 1.023578 & 1.029635 & 1.029638 & 1.029639 \\
.9 & .000000 & .900000 & 1.243692 & 1.260138 & 1.260157 & 1.260158 \\
.99 & .000000 & .990000 & 1.484940 & 1.523572 & 1.523675 & 1.523677 \\
\hline
\end{tabular}

TABLE 2.

The convergence of $u_{n}(x)$ to $u(x)=\tan x, u_{0}(x)=x+x^{3} / 3$.

\begin{tabular}{c|c|c|c|c}
\hline$x$ & $v_{0}(x)$ & $u_{0}(x)$ & $u_{1}(x)$ & $\tan x$ \\
\hline 0.0 & .000000 & .000000 & .000000 & .000000 \\
.1 & .100333 & .100335 & .100335 & .100335 \\
.2 & .202667 & .202710 & .202710 & .202710 \\
\hline .3 & .309000 & .309336 & .309336 & .309336 \\
.4 & .421333 & .422793 & .422793 & .422793 \\
.5 & .541667 & .546301 & .546302 & .546302 \\
.6 & .672000 & .684127 & .684136 & .684137 \\
.7 & .814333 & .842233 & .842288 & .842288 \\
\hline .8 & .970667 & 1.029369 & 1.029638 & 1.029639 \\
.9 & 1.143000 & 1.258988 & 1.260157 & 1.260158 \\
.99 & 1.313433 & 1.519602 & 1.523673 & 1.523677 \\
\hline
\end{tabular}

of the problem exists, though later we shall prove this. We assume that $f$ is strictly convex as a function of $u$ for all $u$ and $x$ that come into consideration, and, in addition, is continuous in $u$ and $x$ and has a bounded second partial derivative with respect to $u$.

As before, we shall not indicate explicitly the dependence of $f$ on $x$ and shall indicate partial derivatives with respect to $u$ by primes.

In view of the assumed convexity we write equation (4.1) in the form

$$
u^{\prime \prime}=\operatorname{Max}\left[f(v)+(u-v) f^{\prime}(v)\right],
$$


where the maximization is over all functions $v$ defined on the interval $[0, b]$. Then we introduce the function $w=w[x ; v]$ which is defined to be the solution of the associated linear boundary value problem

$$
w^{\prime \prime}=f(v)+(w-v) f^{\prime}(v), \quad w(0)=w(b)=0 .
$$

We wish to show first that $u$ may be represented in the form

$$
u(x)=\operatorname{Min}_{v} w[x ; v], \quad 0 \leqq x \leqq b,
$$

provided that $b$ is sufficiently small.

Representation Theorem. For any choice of the function $v$ we may write equation (4.3) in the form

$$
u^{\prime \prime}=f(v)+(u-v) f^{\prime}(v)+p
$$

where $p$ is a non-negative function. Upon introducing the difference function

$$
z=u-w
$$

it is seen that $z$ satisfies the equation

$$
z^{\prime \prime}-f^{\prime}(v) z=p, \quad z(0)=z(b)=0 .
$$

We now have to establish that $z$ is non-positive, whatever $v$, in the sufficiently small interval $[0, b], p$ being non-negative. Essentially this means that we must establish that the Green's function for the operator $d^{2} / d x^{2}-f^{\prime}(v)$ on the interval $[0, b]$, zero boundary values being imposed, does not change sign, under suitable conditions on $f^{\prime}(v)$.

In the event that $f^{\prime}(v)$ is greater than zero for all $v$, the proof can be given simply by contradiction. Suppose $z$ to be positive within the interval so that it has a positive maximum at an interior point of the interval $[0, b]$. Then at this point

$$
z^{\prime \prime}=p+f^{\prime}(v) z
$$

which is greater than zero. This stands in contradiction to the fact that at an interior maximum point $z^{\prime \prime}$ must be non-positive. We shall, however, establish a stronger result which does not require that $f^{\prime}(v)$ be positive. We do this by establishing the following lemma, using a proof due to Bellman, [8]. The lemma is also contained in recent work of AronszaJn \& SMITH, [1, 2], on the connection between reproducing kernels and Green's functions.

Lemma 4.1. Let the solution of the inhomogeneous equation

$$
u^{\prime \prime}+q(x) u=f(x), \quad u(0)=u(b)=0,
$$

be given by

$$
u=\int_{0}^{b} K(x, y) f(y) d y
$$


where $K(x, y)$ is the Green's function for the problem of the equation (4.10). If $q(x)$ satisfies the condition

$$
q(x) \leqq\left(\frac{\pi}{b}\right)^{2}-d, \quad d>0,
$$

where $(\pi / b)^{2}$ appears as the smallest characteristic value of the Sturm-Liouville problem

$$
u^{\prime \prime}+\lambda u=0, \quad u(0)=u(b)=0
$$

then

$$
K(x, y) \leqq 0, \quad 0 \leqq x, y \leqq b .
$$

Proof. Consider the problem of minimizing $J[u]$, where

$$
J[u]=\int_{0}^{b}\left[\left(u^{\prime}\right)^{2}-q(x) u^{2}+2 f u\right] d x
$$

under the assumption concerning $q(x)$ stated in condition (4.12). The minimization is over all functions $u$ for which the integral exists and for which $u(0)=$ $u(b)=0$. The condition in (4.12) asserts the positive definite nature of the quadratic functional $\int_{0}^{b}\left[\left(u^{\prime}\right)^{2}-q(x) u^{2}\right] d x$, which ensures the existence of a minimum of $J[u]$. The minimizing function is determined by the Euler equation of $J[u]$, which is precisely equation (4.10).

A necessary and sufficient condition that $K(x, y)$ be non-positive is that $u(x)$, as given by equation (4.11), be non-positive for $0 \leqq x \leqq b$, whenever $f(x)$ is non-negative in that interval.

Assuming $f(x) \geqq 0$, let us show $u(x) \leqq 0$ for $0 \leqq x \leqq b$. Suppose that $u(x)$ were positive in some interval $\left[x_{0}, x_{1}\right]$. Then by redefining $u(x)$ to be $-u(x)$ in this interval, but otherwise the same, we would leave unchanged the values of the quadratic terms in $J[u]$, but would decrease the value of $\int_{0}^{b} 2 f u d x$, which contradicts the assumption that $u$ minimizes $J[u]$. The proposed changes in $u(x)$ may introduce discontinuities in $u^{\prime}(x)$, but they do not influence the integrability of $\left(u^{\prime}\right)^{2}$ or of $q(x) u^{2}$.

On the basis of the lemma just established, we may now state that if

$$
f^{\prime}(v) \geqq-\left(\frac{\pi}{b}\right)^{2}+d, \quad d>0,
$$

then

$$
z=u-w \leqq 0, \quad 0 \leqq x \leqq b .
$$

In addition, we observe that if $v$ is chosen equal to $u$ in equation (4.4) then

$$
w=w[x ; u]=u .
$$

This completes the proof of the representation (4.5) which we now state as 
Theorem 4.1. Let us assume that $f(u)$ is strictly convex for all finite $u$ and that $f^{\prime \prime}(u)$ exists and is bounded for all finite $u$. Also let us assume that

$$
f^{\prime}(u) \geqq-\left(\frac{\pi}{b}\right)^{2}+d, \quad d>0,
$$

for all finite $u$. Then, for $b$ sufficiently small, which guarantees a unique solution of equations (4.1) and (4.2), we can assert that

$$
u=\operatorname{Min}_{v} w[x ; v],
$$

where $w$ is the solution of the associated linear equation (4.4). The minimum is actually attained for $v=u$.

Remark. The restriction that $f^{\prime}(u) \geqq-(\pi / b)^{2}+d, d>0$, for all finite $u$ can be reduced to the weaker assumption that this inequality hold for functions $u=u(x)$ satisfying a constraint of the form $|u| \leqq m_{1}$, where $m_{1}$ is an $a$ priori bound on the solution of equations (4.1) and (4.2), established, for example, in the course of an existence proof using the Picard method of successive approximations. This means that the convexity and regularity properties of $f(u)$ need hold only for $|u| \leqq m_{1}$. The restricted representation is then

$$
u=\operatorname{Min}_{|v| \leqq m_{1}} w[x ; v] .
$$

Corollaries. It is clear that the function $w$ determined by the choice $v=v_{1}$ provides a uniform upper bound on $u$ :

$$
u \leqq w\left[x ; v_{1}\right] .
$$

Below we shall see that a sequence $\left\{u_{n}\right\}$ of upper bounds converging monotonically to $u$ can be obtained.

If we let

$$
w=\int_{0}^{b} G(x, y)\left[f(v)-v f^{\prime}(v)\right] d y
$$

where $G(x, y)$ is the non-positive Green's function for the operator $d^{2} / d x^{2}-f^{\prime}(v)$ on the interval $[0, b]$, for zero boundary conditions, then $u$ satisfies the relation

$$
u(x)=\int_{0}^{b} G(x, y)\left[f(u(y))-u(y) f^{\prime}(u(y))\right] d y .
$$

Newtonian Approximation. Using the same line of reasoning as in Sections 1 and 3 we are led to introduce the sequence of functions $\left\{u_{n}(x)\right\}, 0 \leqq x \leqq b$, $n=0,1,2, \cdots$, defined by the recurrence relations

$$
\begin{gathered}
u_{0}^{\prime \prime}=f\left(v_{0}\right)+f^{\prime}\left(v_{0}\right)\left(u_{0}-v_{0}\right), \quad u_{0}(0)=u_{0}(b)=0, \\
u_{n+1}^{\prime \prime}=f\left(u_{n}\right)+f^{\prime}\left(u_{n}\right)\left(u_{n+1}-u_{n}\right), \\
u_{n+1}(0)=u_{n+1}(b)=0, \quad n=0,1,2, \cdots .
\end{gathered}
$$


As we shall see, the sequence $\left\{u_{n}(x)\right\}$ forms a bounded monotone decreasing sequence of functions which converge to $u(x)$, the solution of equations (4.1) and (4.2). The convergence is sufficiently rapid so that computation of the sequence $\left\{u_{n}\right\}$ offers a new tool for the numerical integration of two-point boundary value problems, a point which will be illustrated below by an example.

First let us demonstrate the uniform boundedness of the sequence $\left\{u_{n}\right\}$ on a sufficiently small common interval $[0, b]$. We define, as earlier,

$$
\left\|u_{n}(x)\right\|=\operatorname{Max}_{0 \leqq x \leqq b}\left|u_{n}(x)\right|, \quad n=0,1,2, \cdots,
$$

and we choose an initial function $v_{0}(x)$ so that

$$
\underset{0 \leqq x \leqq b}{\operatorname{Max}}\left|v_{0}(x)\right|=\left\|v_{0}(x)\right\| \leqq 1 .
$$

Then $u_{0}(x)$ satisfies the integral equation

$$
u_{0}(x)=\int_{0}^{b} K(x, y)\left[f\left(v_{0}(y)\right)+f^{\prime}\left(v_{0}(y)\right)\left(u_{0}(y)-v_{0}(y)\right)\right] d y,
$$

where the Green's function $K(x, y)$ is given by

$$
K(x, y)=\left[\begin{array}{ll}
(y / b-1) x, & x<y \\
(x / b-1) y, & x>y
\end{array}\right.
$$

From this, it is seen that

$$
\operatorname{Max}_{0 \leqq x, y \leqq b}|K(x, y)|=\frac{1}{4} b .
$$

This, in turn, implies that

$$
\left\|u_{0}\right\| \leqq \frac{1}{4} b^{2} m\left\{2+\left\|u_{0}\right\|\right\}
$$

where for $\left|v_{0}\right| \leqq 1$

$$
m=\operatorname{Max}\left\{\left|f\left(v_{0}(y)\right)\right|,\left|f^{\prime}\left(v_{0}(y)\right)\right|\right\}
$$

Consequently

$$
\left\|u_{0}\right\| \leqq \frac{\frac{1}{2} b^{2} m}{1-\frac{1}{4} b^{2} m} \leqq 1
$$

for $b^{2}$ chosen less than $4 / 3 m$. Proceeding by induction, we assume that for some $n$ we have

$$
\left\|u_{n}\right\| \leqq 1, \quad 0 \leqq x \leqq b<\sqrt{\frac{4}{3 m}}
$$

Then from equation (4.25) we have

$$
u_{n+1}(x)=\int_{0}^{x} K(x, y)\left[f\left(u_{n}(y)\right)+f^{\prime}\left(u_{n}(y)\right)\left(u_{n+1}(y)-u_{n}(y)\right)\right] d y
$$


where $K(x, y)$ is the Green's function defined above. As before this yields

$$
\left\|u_{n+1}(x)\right\| \leqq \frac{\frac{1}{2} b^{2} m}{1-\frac{1}{4} b^{2} m} \leqq 1
$$

for $0 \leqq x \leqq b<\sqrt{4 / 3 m}$. This completes the induction and establishes a common interval of boundedness for the sequence $\left\{u_{n}(x)\right\}$.

We now turn to the question of the monotone decreasing character of the sequence $\left\{u_{n}\right\}$. From equation (4.25) we see that

$$
\begin{aligned}
u_{n+1}^{\prime \prime} & =f\left(u_{n}\right)+f^{\prime}\left(u_{n}\right)\left(u_{n+1}-u_{n}\right), \\
u_{n}^{\prime \prime} & =f\left(u_{n-1}\right)+f^{\prime}\left(u_{n-1}\right)\left(u_{n}-u_{n-1}\right),
\end{aligned}
$$

with the common boundary conditions $u_{k}(0)=u_{k}(b)=0, k=0,1,2, \cdots$. It then follows from the convexity of $f(v)$ ( $c f$. the argument in Section 3 ) that

$$
u_{n}^{\prime \prime} \leqq f\left(u_{n}\right)+f^{\prime}\left(u_{n}\right)\left(u_{n}-u_{n}\right) \text {, }
$$

which implies that

$$
\left(u_{n+1}-u_{n}\right)^{\prime \prime} \geqq f^{\prime}\left(u_{n}\right)\left(u_{n+1}-u_{n}\right)
$$

for $0 \leqq x \leqq b, n=0,1,2, \cdots$. Introducing the function $d_{n+1}$,

$$
d_{n+1}=u_{n+1}-u_{n}
$$

we see that

$$
d_{n+1}^{\prime \prime}=f^{\prime}\left(u_{n}\right) d_{n+1}+p, \quad d_{n+1}(0)=d_{n+1}(b)=0,
$$

where $p$ is non-negative. From the lemma proved above, we deduce, for $b$ sufficiently small, that

$$
d_{n+1}(x) \leqq 0 ;
$$

that is, the sequence $\left\{u_{n}\right\}$ is monotone decreasing (actually monotone nonincreasing) for $n=0,1, \cdots$.

Since the sequence $\left\{u_{n}\right\}$ is monotone decreasing and uniformly bounded it converges to a limit function $U(x), 0 \leqq x \leqq b$. Using Lebesgue's theorem on bounded convergence and letting $n$ become infinite in equation (4.35), we see that $U(x)$ satisfies the integral equation

$$
U(x)=\int_{0}^{b} K(x, y) f(U(y)) d y .
$$

This establishes that $U(x)$ is the solution of equations (4.1) and (4.2), and thus that

$$
U(x)=u(x), \quad 0 \leqq x \leqq b .
$$

We have now completed the proof of

Theorem 4.2. The sequence $\left\{u_{n}\right\}$ defined by equation (4.25) converges mono- 
tonically to the solution of equations (4.1) and (4.2) in a sufficiently small interval $0 \leqq x \leqq b$.

Quadratic Convergence. We shall now extend the above result by showing that

$$
\left\|d_{n+1}\right\| \leqq k\left\|d_{n}\right\|^{2}
$$

where, we recall,

$$
d_{n+1}(x)=u_{n+1}(x)-u_{n}(x),
$$

and $k$ is a constant independent of $n$ for $x$ in a sufficiently small interval $[0, b]$. From equation (4.37) we see that

$$
d_{n+1}^{\prime \prime}=\left[f\left(u_{n}\right)-f\left(u_{n-1}\right)-d_{n} f^{\prime}\left(u_{n-1}\right)\right]+f^{\prime}\left(u_{n}\right) d_{n+1}, \quad n=1,2,3, \cdots .
$$

Then, using the Green's function introduced in equation (4.29) and the mean value theorem, we find

$$
d_{n+1}(x)=\int_{0}^{b} K(x, y)\left[\frac{1}{2} f^{\prime \prime}\left(\bar{q}_{n}(y)\right) d_{n}^{2}+f^{\prime}\left(u_{n}(y)\right) d_{n+1}(y)\right] d y
$$

where $\bar{q}_{n}(x)$ lies between $u_{n}(x)$ and $u_{n-1}(x)$ for $0 \leqq x \leqq b$. Using the previously demonstrated uniform boundedness of the sequence $\left\{u_{n}(x)\right\}$ on $[0, b]$, and the assumptions concerning $f(v)$, let us write

$$
m=\operatorname{Max}_{|v| \leqq 1}\left\{|f(v)|,\left|f^{\prime}(v)\right|, \frac{1}{2}\left|f^{\prime \prime}(v)\right|\right\},
$$

from which it follows that

$$
\left\|d_{n+1}\right\| \leqq \frac{1}{4} b^{2}\left[m\left\|d_{n}\right\|^{2}+m\left\|d_{n+1}\right\|\right]
$$

or

$$
\left\|d_{n+1}\right\| \leqq \frac{b^{2} m}{4-b^{2} m}\left\|d_{n}\right\|^{2}
$$

which establishes the assertion.

In addition, it is clear that this establishes the uniform convergence of the sequence of continuous functions $u_{n}$ to the limit $V(x)$ on the sufficiently small interval $[0, b]$. Passing to the limit in equation (4.35) we then find that

$$
V(x)=\int_{0}^{b} K(x, y) f(V(y)) d y
$$

from which it follows that $V(x)$ satisfies equations (4.1) and (4.2).

Lastly, we point out that in a similar manner we can establish that

$$
\left\|u-u_{n}\right\| \leqq k_{1}\left\|u-u_{n-1}\right\|^{2}, \quad n=1,2, \cdots,
$$

where $k_{1}$ is independent of $n$, which establishes the quadratic convergence of the sequence $\left\{u_{n}\right\}$. 
Numerical Example. As an illustration of the approach developed in the previous paragraphs, let us consider the nonlinear two-point boundary value problem

$$
u^{\prime \prime}=e^{u}, \quad u(0)=u(b)=0,
$$

which is of current interest in connection with some problems in electro-hydrodynamics (see KELLER, [23, 24]) and the physics of semiconductors. The standard approach to the numerical solution is to use the condition $u(0)=0$ and then approximate the initial slope $u^{\prime}(0)$ which will result in $u(1)=0$.

Our approach is radically different. We approximate the function $u(x)$ by the sequence of functions $u_{n}(x)$ defined by the linear recurrence relations

$$
\begin{aligned}
u_{0}^{\prime \prime} & =e^{v_{0}}+e^{v_{0}}\left(u_{0}-v_{0}\right), & u_{0}(0) & =u_{0}(b)=0, \\
u_{n+1}^{\prime \prime} & =e^{u_{n}}+e^{u_{n}}\left(u_{n+1}-u_{n}\right), & u_{n+1}(0) & =u_{n+1}(b)=0, \quad n=0,1, \cdots,
\end{aligned}
$$

where $v_{0}(x)$ is the initial approximation function. We have now replaced the original nonlinear two-point boundary value problem with the problem of computing a sequence of solutions of linear equations with two-point boundary conditions. This is an important simplification, and especially so when coupled with the fact that the sequence is quadratically convergent.

Assuming that $u_{n}(x)$ has been determined, we determine $u_{n+1}(x)$ in terms of the appropriate linear combination of solutions of the homogeneous and inhomogeneous equations. Thus we introduce the function $y_{n+1}(x)$ and $z_{n+1}(x)$ defined by

$$
y_{n+1}^{\prime \prime}-e^{u_{n}} y_{n+1}=0, \quad y_{n+1}(0)=0, \quad y_{n+1}^{\prime}(0)=1, \quad n=0,1,2, \cdots,
$$

and

$$
z_{n+1}^{\prime \prime}-e^{u_{n}} z_{n+1}=e^{u_{n}}\left(1-u_{n}\right), \quad z_{n+1}^{\prime}(0)=0, \quad n=0,1,2, \cdots .
$$

In terms of these functions $u_{n+1}(x)$ is given by the expression

$$
u_{n+1}(x)=-\frac{z_{n+1}(b)}{y_{n+1}(b)} y_{n+1}(x)+z_{n+1}(x)
$$

from which we see that the boundary conditions are both fulfilled for $n=$ $0,1,2, \cdots$. The initial slope of $u_{n+1}(x)$ is seen to be $-z_{n+1}(b) / y_{n+1}(b)$. This means that $u_{n+1}(x)$ may also be determined, as a numerical check, as the solution of the initial value problem

$$
u_{n+1}^{\prime \prime}-e^{u_{n}} u_{n+1}=e^{u_{n}}\left(1-u_{n}\right), \quad u_{n+1}(0)=0, \quad u_{n+1}^{\prime}(0)=-\frac{z_{n+1}(b)}{y_{n+1}(b)} .
$$

The problem for $b=1$ was computed on the Johnniac using a Runge-Kutta. method of integration capable of yielding at least six accurate figures for $0 \leqq x \leqq 1$. Eight seconds of running time elapsed for each iteration; two iterations yielded six figure accuracy in the approximation of $u_{n}(x)$ to $u_{6}(x)$.

The explicit solution of the problem of equation (4.54) is 


$$
u(x)=-\ln 2+2 \ln \{c \sec 0.5 c(x-0.5)\},
$$

where $c$ is the root of the transcendental equation

$$
\sqrt{2}=c \sec (0.25 c)
$$

which lies between zero and $\frac{1}{2} \pi$. Using Newton's method, $c$ was determined to be $c=1.3360557$.

The results of a computation in which $v_{0}$ was chosen to be identically zero are displayed in Table 3 , in which the digits of $u_{n}(x)$ in agreement with those of $u(x)$ are in italics. As expected, the number of digits in agreement approximately doubles with each iteration.

For higher order nonlinear equations the method should be even more advantageous.

TABLE 3.

The approach of $u_{n}(x)$ to $u(x)$.

\begin{tabular}{c|c|c|c|c}
\hline$x$ & $v_{0}(x)$ & $u_{0}(x)$ & $u_{1}(x)$ & $u(x)$ \\
\hline 0.0 & .000000 & .000000 & .000000 & .000000 \\
0.1 & .000000 & -.041285 & -.041436 & -.041436 \\
0.2 & .000000 & -.072974 & -.0732683 & -.0732686 \\
0.3 & .000000 & -.095386 & -.095800 & -.095800 \\
0.4 & .000000 & -.108743 & -.109238 & -.109238 \\
0.5 & .000000 & -.119181 & -.113904 & -.113704 \\
0.6 & .000000 & -.108743 & -.109238 & -.109238 \\
0.7 & .000000 & -.095386 & -.095800 & -.095800 \\
0.8 & .000000 & -.072974 & -.0732683 & -.0732686 \\
0.9 & .000000 & -.041285 & -.041436 & -.041436 \\
1.0 & .000000 & .000000 & .000000 & .000000 \\
\hline
\end{tabular}

5. The Equation $u^{\prime \prime}=f\left(u^{\prime}, u, x\right), u(0)=u(b)=0$. Our purpose in this section is to show that the equation

$$
u^{\prime \prime}=f\left(u^{\prime}, u, x\right), \quad u(0)=u(b)=0,
$$

under the assumptions that the function $f$ is strictly convex in $u^{\prime}$ and $u$ and is sufficiently smooth for all values of its arguments that come under consideration, can be treated using the same techniques outlined above. In fact, we reduce this problem to that treated in Section 4.

Representation Theorem. In view of the assumption that $f$ is convex in $u^{\prime}$ and $u$ (see Section 2) we may write equation (5.1) in the form

$$
u^{\prime \prime}=\operatorname{Max}_{v_{1}, v}\left\{f\left(v_{1}, v\right)+\left(u^{\prime}-v_{1}\right) f_{v_{1}}+(u-v) f_{v}\right\},
$$

where as usual we do not explicitly indicate the dependence of $f$ on the in- 
dependent variable $x$. The maximum is attained for $v_{1}=u^{\prime}$ and $v=u$. From equation (5.2) it follows that

$$
u^{\prime \prime}=f\left(v_{1}, v\right)+\left(u^{\prime}-v_{1}\right) f_{v_{1}}+(u-v) f_{v}+p,
$$

where $p$ is non-negative for any choice of the functions $v_{1}$ and $v$. Our aim is to show that $u$, assumed to exist, is given by

$$
u=\operatorname{Min}_{v_{1}, v} w\left[x ; v_{1}, v\right], \quad 0 \leqq x \leqq b,
$$

where $w=w\left[x ; v_{1}, v\right]$ is the solution of the associated linear equation

$$
w^{\prime \prime}=f\left(v_{1}, v\right)+\left(w^{\prime}-v_{1}\right) f_{v_{1}}+(w-v) f_{v}, \quad w(0)=w(b)=0,
$$

provided that the interval $[0, b]$ is sufficiently small.

The difference function

$$
z=u-w
$$

satisfies the equation

$$
z^{\prime \prime}=z^{\prime} f_{v_{1}}+z f_{v}+p
$$

or

$$
z^{\prime \prime}-f_{v_{1}} z^{\prime}-f_{v} z=p, \quad z(0)=z(b)=0 .
$$

We wish to demonstrate that $z$ is non-positive in the sufficiently small interval $[0, b]$. We do this by eliminating the term involving $z^{\prime}$ through use of the well known transformation

$$
z_{1}=z \exp \left\{-\frac{1}{2} \int_{0}^{x} f_{v_{1}}\left(v_{1}(t), v(t)\right) d t\right\} .
$$

The function $z_{1}$ has the same sign as $z$ and satisfies the same homogeneous boundary conditions. Under the substitution (5.9) the equation (5.7) becomes

$$
\begin{gathered}
z_{1}^{\prime \prime}+\left[\frac{1}{2} \frac{d}{d x}\left(f_{v_{1}}\right)-f_{v}-\frac{1}{4}\left(f_{v_{1}}\right)^{2}\right] z_{1}=p \exp \left\{-\frac{1}{2} \int_{0}^{x} f_{v_{1}} d t\right\}, \\
z_{1}(0)=z_{1}(b)=0 .
\end{gathered}
$$

By the lemma of Section 4 we see that for the quantity $b$ sufficiently small we have

$$
z_{1}(x) \leqq 0, \quad 0 \leqq x \leqq b .
$$

This carries with it the non-positivity of $z=u-w$ and shows that

$$
u \leqq w\left[x ; v_{1}, v\right], \quad 0 \leqq x \leqq b .
$$

In addition, equality holds if $v_{1}=u^{\prime}$ and $v=u$, as is seen from equations (5.1) and (5.5). Thus we have established the desired representation. 
Theorem 5.1. The solution of equation (5.1) may be represented in the form

$$
u=\operatorname{Min}_{v_{1}, v} w\left[x ; v_{1}, v\right], \quad 0 \leqq x \leqq b,
$$

provided that $b$ is sufficiently small.

An immediate corollary is that $w\left[x ; v_{1} v\right]$, where $v_{1}$ and $v$ are arbitrary, provides an upper bound for $u$ in the interval $[0, b]$.

Newtonian Approximation. Following our earlier discussions, we introduce the sequence of functions $\left\{u_{n}(x)\right\}$ by the relations

$$
\begin{gathered}
u_{0}^{\prime \prime}=f\left(v_{0}^{\prime}, v_{0}\right)+f_{u^{\prime}}\left(v_{0}^{\prime}, v_{0}\right)\left(u_{0}^{\prime}-v_{0}^{\prime}\right)+f_{u}\left(v_{0}^{\prime}, v_{0}\right)\left(u_{0}-v_{0}\right), \\
u_{n+1}^{\prime \prime}=f\left(u_{n}^{\prime}, u_{n}\right)+f_{u^{\prime}}\left(u_{n}^{\prime}, u_{n}\right)\left(u_{n+1}^{\prime}-u_{n}^{\prime}\right)+f_{n}\left(u_{n}^{\prime}, u_{n}\right)\left(u_{n+1}-u_{n}\right), \\
u_{n}(0)=u_{n}(b)=0
\end{gathered}
$$

which hold for $n=0,1,2, \cdots$.

The discussions of the monotonicity and quadratic convergence parallel those of the previous sections, mutatis mutandis. Let us now display the results of a numerical experiment.

Numerical Example. The equation

$$
-u^{\prime \prime}=1+\alpha^{2}\left(u^{\prime}\right)^{2}, \quad u(0)=u(b)=0,
$$

suggested by Professor Prager, arises in connection with finite deflections of an elastic string under a transverse load. The problem for which $\alpha^{2}=0.49$ and $b=1$ was treated by determining numerically the sequence of approximations $\left\{u_{n}(x)\right\}$ given by the linear recurrence relations

$$
\begin{gathered}
-u_{0}^{\prime \prime}=1-0.49\left(v_{0}^{\prime}\right)^{2}+2(0.49) v_{0}^{\prime} u_{0}^{\prime}, \quad u_{0}(0)=u_{0}(1)=0 \\
-u_{n+1}^{\prime \prime}=1-0.49\left(u_{n}^{\prime}\right)^{2}+2(0.49) u_{n}^{\prime} u_{n+1}^{\prime}, \\
n=0,1,2, \cdots .
\end{gathered}
$$

As before, to compute $u_{n+1}(x)$, given $u_{n}(x)$, we first compute solutions of appropriate initial value problems for the homogeneous and inhomogeneous equations as follows:

$$
\begin{aligned}
& y_{n+1}^{\prime \prime}+2(0.49) u_{n}^{\prime} y_{n+1}^{\prime}=0, \quad y_{n+1}^{\prime}(0)=0, \quad y_{n+1}^{\prime}(0)=1, \\
& z_{n+1}^{\prime \prime}+2(0.49) u_{n}^{\prime} z_{n+1}^{\prime}=0.49\left(u_{n}^{\prime 2}\right)-1, \quad z_{n+1}(0)=z_{n+1}^{\prime}(0)=0
\end{aligned}
$$

for $n=0,1,2, \cdots$. Then for $u_{n+1}(x)$ we have

$$
u_{n+1}(x)=-\frac{z_{n+1}(1)}{y_{n+1}(1)} y_{n+1}(x)+z_{n+1}(x)
$$

As initial approximations, $v_{0}(x)=v_{0}^{\prime}(x)=0$ are used. 
Using a numerical procedure yielding $u_{n}(x)$ correct to five figures, each iteration consuming about eight seconds of running time on the Johnniac, the data in Table 4 were obtained. Two iterations provide five figure accuracy. The closed form solution of equation (5.15) is

$$
u(x)=\frac{1}{\alpha^{2}} \ln \frac{\cos \alpha(x-0.5)}{\cos 0.5 \alpha},
$$

which is given in the last column. The digits of $u_{n}(x)$ in agreement with those of $u(x)$ are in italics.

TABLE 4.

The convergence of $u_{n}(x)$ to $u(x)$.

\begin{tabular}{c|c|c|c|c}
\hline$x$ & $v_{0}(x)$ & $u_{0}(x)$ & $u_{1}(x)$ & $u(x)$ \\
\cline { 2 - 4 } 0.0 & .000000 & .000000 & .000000 & .000000 \\
.1 & .000000 & .045000 & .046570 & .046571 \\
.2 & .000000 & .080000 & .082302 & .082304 \\
\hline .3 & .000000 & .105000 & .107571 & .107573 \\
.4 & .000000 & .120000 & .122632 & .122635 \\
.5 & .000000 & .125000 & .127636 & .127639 \\
.6 & .000000 & .120000 & .122632 & .122635 \\
.7 & .000000 & .105000 & .107571 & .107573 \\
\hline .8 & .000000 & .080000 & .082302 & .082304 \\
.9 & .000000 & .045000 & .046570 & .046571 \\
1.0 & .000000 & .000000 & .000000 & .000000 \\
\hline
\end{tabular}

\section{Partial Differential Equations}

6. The Equation $u_{x x}+u_{y y}=f(u, x, y)$. Let us now consider how we may treat the nonlinear partial differential equation of elliptic type

$$
\begin{aligned}
u_{x x}+u_{y y} & =f(u, x, y), \quad(x, y) \varepsilon D, \\
u & =\bar{u}, \quad(x, y) \varepsilon B,
\end{aligned}
$$

where $B$ is the boundary of the domain $D$ in the $x, y$-plane. In particular, we wish to obtain a representation for the solution of equation (6.1) in terms of the maximum operation applied to the solution of an associated linear equation, which we shall then use to obtain a monotone sequence of functions which converges to $u$.

We assume that $f(u, x, y)$ is a strictly convex function of $u$ for all finite $u$ and for all $(x, y)$ in the domain $D$, the boundary $B$ of which is assumed to have a continuously turning tangent. In addition, it is to have a continuous second partial derivative with respect to $u$ and is to be continuous as a function of its 
three arguments. As usual, we shall not indicate the explicit dependence of $f$ on $x$ and $y$ and shall indicate partial derivatives of $f$ with respect to $u$ by primes.

Let us assume that $\bar{u}$ is continuous on the boundary, and let $u_{1}$ be the potential function which assumes the given boundary values $\bar{u}$ on $B$. Then the difference function $u_{2}=u-u_{1}$ satisfies the differential equation and boundary condition

$$
\begin{gathered}
\Delta u_{2}=\Delta u=f(u)=f\left(u_{1}+u_{2}\right)=g\left(u_{2}\right), \\
u_{2}=0, \quad(x, y) \varepsilon B,
\end{gathered}
$$

where $g\left(u_{2}\right)$ is strictly convex and $\Delta$ is the Laplace operator. We shall therefore limit ourselves to the problem

$$
\begin{aligned}
\Delta u & =f(u), & & (x, y) \varepsilon D, \\
u & =0, & & (x, y) \varepsilon B
\end{aligned}
$$

(cf. Courant-Hilbert, [15], V. 2, pp. 286-288).

Special cases of equations (6.1) and (6.3), for which $f(u)=e^{u}$, as we have remarked, are of interest in studies of electrohydrodynamics, [23, 24], and the physics of semiconductors. This equation is also of historical interest, for this equation was treated by PICARD in his classic paper on the method of successive approximations referred to earlier.

Representation. We may write, according to Section 2, equation (6.3) in the form

$$
\begin{aligned}
\Delta u & =\operatorname{Max}_{v}\left[f(v)+(u-v) f^{\prime}(v)\right], \\
u & =0, \quad(x, y) \varepsilon B,
\end{aligned}
$$

where the maximization is over all functions $v$ defined on the domain $D$. The maximum occurs for $v=u$. Consequently we may write

$$
\Delta u=f(v)+(u-v) f^{\prime}(v)+p,
$$

where $p$ is non-negative, whatever the choice of $v$. If we introduce $w=w[x, y ; v]$ as the solution of the associated linear equation

$$
\begin{aligned}
\Delta w & =f(v)+(w-v) f^{\prime}(v), \\
w & =0, \quad(x, y) \varepsilon B,
\end{aligned}
$$

which we assume determines a unique function $w$ for each admissible function $v$, then the difference function

$$
z=u-w
$$

satisfies the equation

$$
\begin{gathered}
\Delta z-f^{\prime}(v) z=p, \\
z=0, \quad(x, y) \varepsilon B .
\end{gathered}
$$


We wish now to show that $z$ is non-positive under the assumptions that $p$ is non-negative throughout the domain $D$. To do this we make use of the following lemma, which is the analogue of the lemma of Section 4:

Lemma 6.1. If

and if

$$
\begin{gathered}
\Delta u+q(x, y) u=p \geqq 0, \quad(x, y) \varepsilon D \\
u=0, \quad(x, y) \varepsilon B
\end{gathered}
$$

$$
q(x, y) \leqq \lambda_{1}-d, \quad d>0,
$$

where $\lambda_{1}$ is the smallest characteristic value of the problem

$$
\begin{aligned}
\Delta u+\lambda u & =0, & & (x, y) \varepsilon D, \\
u & =0, & & (x, y) \varepsilon B,
\end{aligned}
$$

then $u$ is non-positive on the domain $D$.

Proof. The proof parallels that of the lemma of Section 4. In essence, this lemma provides us with a statement to the effect that the Green's function for the operator $\Delta+q$ does not change sign in $D$, zero boundary values being assumed.

By applying this lemma to equation (6.8) we see that if $f^{\prime}(v) \geqq-\lambda_{1}+d$, $d>0$, then $z$ is non-positive on the domain $D$. This implies that $u$ does not exceed $w$ on $D$. In addition, the solution of equation (6.6) for $v=u$ is precisely $w=u$. This completes the demonstration of

Theorem 6.1. The solution of equation (6.3) on the domain D may be represented in the form

$$
u=\operatorname{Min} w[x, y ; v], \quad(x, y) \varepsilon D,
$$

where $w$ is the solution of equation (6.6), provided that the area of the domain $D$ is sufficiently small to guarantee that

$$
f^{\prime}(v) \geqq-\lambda_{1}+d, \quad d>0 .
$$

It follows that upper bounds on the solution $u$ are readily obtained.

In the applications it is important to realize that the restrictions $f^{\prime \prime}(v)>0$ and $f^{\prime}(v) \geqq-\lambda_{1}+d$ need not be fulfilled for all $v$, but only for those $v$ in a neighborhood of the solution $u$. As a result, e.g., of a proof using successive approximations, we may know that in the sufficiently small domain $D$ the solution $u$ of equation (6.3) is bounded from above and below so that $m_{1} \leqq u \leqq m_{2}$. Since the minimizing value of $v$ is $u$ itself, we may conduct the minimization solely over those functions $v$ for which $m_{1} \leqq v \leqq m_{2}$. This leads to the restricted representation

$$
u=\operatorname{Min}_{m_{1} \leqq v \leqq m_{3}} w[x, y ; v],
$$

which we now formulate as 
Theorem 6.2. The solution of equation (6.3) may be represented in the form of equation (6.14) under the assumptions that

(a) $m_{1} \leqq u \leqq m_{2}$,

(b) $f^{\prime \prime}(v)>0, f^{\prime}(v) \geqq-\lambda_{1}+d, d>0, m_{1} \leqq v \leqq m_{2}$,

(c) $D$ is of sufficiently small area.

To illustrate this, note that the function $f(u)=u+a u^{3}, a>0$, does not have a second derivative that is positive for all $u$. However, if we are able to limit ourselves to a region $R$ in which $u$ is known to be positive, then $f(u)$ will be strictly convex. The remaining condition on $f^{\prime}(u)$ is trivially satisfied. Such simple observations markedly expand the range of applicability of these methods.

Newtonian Approximation. Motivated by the same considerations as in earlier sections, we are led to introduce the sequence of functions $\left\{u_{n}(x, y)\right\}$ defined by the linear recurrence relations

$$
\begin{aligned}
\Delta u_{0} & =f\left(v_{0}\right)+\left(u_{0}-v_{0}\right) f^{\prime}\left(v_{0}\right), \\
\Delta u_{n+1} & =f\left(u_{n}\right)+\left(u_{n+1}-u_{n}\right) f^{\prime}\left(u_{n}\right), \quad u_{k}=0, \quad(x, y) \varepsilon B, \\
k & =0,1,2, \cdots,
\end{aligned}
$$

which we expect to be monotone decreasing and quadratically convergent, if the domain $D$ is sufficiently small. First let us show that the sequence is uniformly bounded.

The relations of equation (6.15) lead to the integral equation

$$
u_{n+1}=\iint_{D} K\left(x, y ; x^{\prime}, y^{\prime}\right)\left[f\left(u_{n}\right)+\left(u_{n+1}-u_{n}\right) f^{\prime}\left(u_{n}\right)\right] d x^{\prime} d y^{\prime},
$$

where $K\left(x, y ; x^{\prime}, y^{\prime}\right)$ is the Green's function for the Laplace operator, the domain $D$, and zero boundary conditions. If we now define the norm of a continuous function $u$ on $D$ to be

$$
\|u\|=\operatorname{Max}_{(x, y) \mathcal{e} D+B}|u|,
$$

and if we set

$$
m \geqq \operatorname{Max}_{|v| \leqq 1}\left\{|f(v)|,\left|f^{\prime}(v)\right|, \frac{1}{2}\left|f^{\prime \prime}(v)\right|\right\},
$$

then we obtain

$$
\left\|u_{n+1}\right\| \leqq \operatorname{Max}_{(x, y) \varepsilon D} \iint_{D}|K| d x^{\prime} d y^{\prime}\left\{|| u_{n+1} \|+2\right\} m,
$$

provided that

$$
|| u_{n} \| \leqq 1
$$


It follows that

$$
\left\|u_{n+1}\right\| \leqq \frac{2 k m}{1-k m} \leqq 1
$$

where

$$
k=\operatorname{Max}_{(x, y)_{\varepsilon} D} \iint_{D}|K| d x^{\prime} d y^{\prime} .
$$

Here we have assumed that

$$
k m<\frac{1}{3} .
$$

Since $k$ tends to zero with decreasing area of the domain $D$, in spite of the fact that $K$ is unbounded, inequality $(6.22)$ can be attained if the area of $D$ is small enough. Furthermore, this induction may be started with $n=0$, by choosing $\left\|v_{0}\right\| \leqq 1$. Hence the sequence $\left\{u_{n}\right\}$ is uniformly bounded on a sufficiently small domain $D$.

Monotonicity. The proof that the sequence $\left\{u_{n}\right\}$ is monotone decreasing follows the usual lines. From equation (6.15) we have

$$
\begin{aligned}
\Delta u_{n+1} & =f\left(u_{n}\right)+\left(u_{n+1}-u_{n}\right) f^{\prime}\left(u_{n}\right), \\
\Delta u_{n} & =f\left(u_{n-1}\right)+\left(u_{n}-u_{n-1}\right) f^{\prime}\left(u_{n-1}\right) .
\end{aligned}
$$

According to the convexity assumption of $f(v)$, the right hand side of the last equation is maximized for $u_{n-1}=u_{n}$, so that

$$
\Delta u_{n} \leqq f\left(u_{n}\right)+\left(u_{n}-u_{n}\right) f^{\prime}\left(u_{n}\right) .
$$

Consequently we see that

$$
\begin{aligned}
& \Delta\left(u_{n+1}-u_{n}\right) \geqq\left(u_{n+1}-u_{n}\right) f^{\prime}\left(u_{n}\right), \\
& u_{n+1}-u_{n}=0, \quad(x, y) \varepsilon B,
\end{aligned}
$$

which, according to the lemma, implies that

$$
u_{n+1}-u_{n} \leqq 0, \quad(x, y) \varepsilon B+D .
$$

Since the integrable functions $\left\{u_{n}(x, y)\right\}$ are monotone decreasing and bounded from below, they converge to a limit function $U(x, y)$. Though $K\left(x, y ; x^{\prime}, y^{\prime}\right)$ in equation (6.16) is unbounded, it is absolutely integrable on the domain $D$ so that, using Lebesgue's theorem, we may interchange the orders of integrating and, passing to the limit, to obtain

$$
U(x, y)=\iint_{D} K\left(x, y ; x^{\prime}, y^{\prime}\right) f\left(U\left(x^{\prime}, y^{\prime}\right)\right) d x^{\prime} d y^{\prime}
$$


Quadratic Convergence. Though the proof of the quadratic convergence of the sequence $\left\{u_{n}\right\}$ is abstractly equivalent to what has been given before, we include it for the sake of completeness. From equations (6.3) and (6.15) we find, upon integrating,

$$
u-u_{n+1}=\iint_{D} K\left[f(u)-f\left(u_{n}\right)-\left(u_{n+1}-u_{n}\right) f^{\prime}\left(u_{n}\right)\right] d x^{\prime} d y^{\prime},
$$

from which it follows, by use of the mean value theorem, that

$$
u-u_{n+1}=\iint_{D} K\left[\frac{1}{2} f^{\prime \prime}\left(\bar{q}_{n}\right)\left(u-u_{n}\right)^{2}+\left(u-u_{n+1}\right) f^{\prime}\left(u_{n}\right)\right] d x^{\prime} d y^{\prime},
$$

where $\bar{q}_{n}$ lies between $u_{n}$ and $u,(x, y) \varepsilon D$. This implies that

$$
\left\|u-u_{n+1}\right\| \leqq k m\left\{|| u-u_{n}\left\|^{2}+\right\| u-u_{n+1} \|\right\},
$$

or

$$
\left\|u-u_{n+1}\right\| \leqq \frac{k m}{1-k m}\left\|u-u_{n}\right\|^{2} .
$$

Hence the sequence $\left\{u_{n}\right\}$ is a quadratically convergent sequence and for $\mathrm{km}<\frac{1}{2}$ is certainly uniformly convergent on $D$. Suppose the limit function to be $V(x, y)$. Making use of the uniform convergence and passing to the limit in equation (6.16) we find that

$$
V=\iint_{D} K f(V) d x^{\prime} d y^{\prime}
$$

Comparisons. It is interesting to note that the sequence of functions $\left\{v_{n}\right\}$, introduced in Courant-Hilbert, [15], V. II, pp. 286-287, to establish the existence of a solution of the problem

$$
\begin{aligned}
\Delta u & =e^{u}, & & (x, y) \varepsilon D, \\
u & =f, & & (x, y) \varepsilon B,
\end{aligned}
$$

can be viewed as a modified Newtonian sequence. The sequence is defined by the relations

$$
\begin{gathered}
\Delta v_{n+1}-e^{w} v_{n+1}=e^{w}\left[e^{v_{n}}-v_{n}\right], \\
v_{n+1}=0, \quad(x, y) \varepsilon B,
\end{gathered}
$$

where $w$ is the potential function equal to $f$ on $B$ :

$$
\begin{aligned}
\Delta w & =0, & & (x, y) \varepsilon D, \\
w & =f, & & (x, y) \varepsilon B .
\end{aligned}
$$


The sequence $\left\{v_{n}\right\}$ has as its limit $v=u-w$, where $v$ is the solution of the problem

$$
\begin{aligned}
\Delta v & =e^{w} e^{v}, & & (x, y) \varepsilon D, \\
v & =0, & & (x, y) \varepsilon B .
\end{aligned}
$$

The Newtonian sequence appropriate for this problem is

$$
\Delta v_{n+1}=e^{w} e^{v_{n}}+e^{w} e^{v_{n}}\left(v_{n+1}-v_{n}\right), \quad v_{k}=0, \quad(x, y) \varepsilon B .
$$

This may be modified "to keep the slopes of the tangent lines constant during the integrations", [30]. The modified sequence is

$$
\Delta v_{n+1}=e^{w} e^{v_{n}}+e^{w} e^{v_{0}}\left(v_{n+1}-v_{n}\right),
$$

or

$$
\begin{aligned}
\Delta v_{n+1}-e^{w} e^{v_{0}} v_{n+1} & =e^{w} e^{v_{n}}-e^{w} e^{v_{0}} v_{n}=e^{w}\left(e^{v_{n}}-e^{v_{0}} v_{n}\right), \\
v_{k} & =0, \quad(x, y) \varepsilon B,
\end{aligned}
$$

which is the sequence used in Courant-HILBERT for $v_{0}=0$.

Though the sequence of equation (6.35) is monotone, we would not expect it to converge as rapidly as the sequence of equation (6.40). It has the advantage that the same Green's function is used throughout the process.

KeLler, in the papers referred to, has introduced a procedure that has the advantage that it produces functions that are alternately greater and less than the solution. Thus the solution is caught in a pincers movement; in this connection compare WEYL's treatment of BLAsius's equation, [47].

7. The Equation $u_{t}-u_{x x}=f(u, x, t)$. Non-linear parabolic equations of the form

$$
u_{t}-u_{x x}=f(u, x, t)
$$

occur in one-dimensional heat flow problems when radiation is taken into account (cf. Siddiqi, [42], Minakshisundaram, [29]) and in various neutron multiplication problems. We shall consider this equation under our usual assumptions that $f$ is strictly convex as a function of $u$, is continuous in all its arguments, and has a bounded second partial derivative with respect to $u$ for all values of the independent variables which come into consideration. As usual, we shall not indicate the specific dependence of $f$ on $x$ and $t$, and shall denote partial derivatives with respect to $u$ by primes.

Equation (7.1) is assumed to hold in the rectangle $R$ with vertices at $(0,0)$, $(1,0),(1, T)$, and $(0, T)$. On the two vertical sides, $x=0,0 \leqq t \leqq T$, and $x=1,0 \leqq t \leqq T$, and on the lower side $t=0,0 \leqq x \leqq 1$, which we denote collectively by $B$, the function $u$ is to be identically zero. There is little loss in generality in assuming this, as the discussion in Section 6 shows. Existence and uniqueness theorems for the solutions of equation (7.1) and its associated linear 
equation for $w$, with $u=w=0$ on $B$ are well known, [5, 29, 39, 42, 46]. Let us first establish the positivity property for the operator

$$
H-f^{\prime}(v) \equiv \frac{\partial}{\partial t}-\frac{\partial^{2}}{\partial x^{2}}-f^{\prime}(v)
$$

which we shall need in our discussion below.

Let us consider the solution of the equation

$$
z_{t}-z_{x x}-f^{\prime}(v) z=p \geqq 0, \quad(x, y) \varepsilon R,
$$

which satisfies the mixed boundary-initial condition

$$
z=0, \quad(x, y) \varepsilon B .
$$

We shall prove the positivity property, under a suitable restriction on $f^{\prime}(v)$. Although the proof is standard, we include it for the sake of completeness.

We shall first assume that

$$
f^{\prime}(v)<0, \quad v<\infty
$$

and shall show that the minimum of $z$ occurs on the boundary $B$, which implies that $z$ is non-negative on $R$. For suppose that $z$ has a minimum in the interior or on the segment $0<x<b, t=T$. Then at this point $z_{t} \leqq 0, z_{x x} \geqq 0$, and $z<0$. This implies there is a point in $R$ for which

$$
z_{t}-z_{x x}-f^{\prime}(v) z<0
$$

which contradicts equation (7.1). Hence the minimum $z$ occurs on $B$ and is zero.

Now let us suppose, as indeed we have, that there exists a constant $m$ such that

$$
f^{\prime}(v)<m, \quad v, m<\infty .
$$

It is still true that $z \geqq 0$. To see this we add and subtract $m z$ from the left hand side of equation (7.3):

$$
z_{t}-z_{x x}-\left(f^{\prime}(v)-m\right) z-m z=p \geqq 0 .
$$

If we now introduce

$$
w=e^{-m t} z
$$

we see that $w$ satisfies the equation

$$
w_{t}-w_{x x}-\left(f^{\prime}(v)-m\right) w=p e^{-m t} \geqq 0,
$$

and is zero on the boundary $B$. Then since $f^{\prime}(v)-m<0$, as above, $w$ is nonnegative, and so is $z$ in the domain $R$.

This completes the proof of the

Lemma 7.1. Let $z$ satisfy the differential inequality

$$
z_{t}-z_{x x}-f^{\prime}(v) z \geqq 0
$$


in the domain $D, 0<x<b, 0<t \leqq T$, and let $z=0$ on the boundary $B, x=0$, $0 \leqq t \leqq T ; x=b, 0<t \leqq T ; 0 \leqq x \leqq b, t=0$. Then, if there is $a$ constant $m$ for $T$ fixed such that

$$
f^{\prime}(v)<m, \quad m<\infty,
$$

in the domain $D, z$ is non-negative throughout the domain $D$.

Representation. The associated linear equation for equation (7.1) is, in terms of $w=w[x, t ; v]$,

$$
w_{t}-w_{x x}-f^{\prime}(v) w=f(v)-v f^{\prime}(v) .
$$

In addition, $w$ satisfies the boundary condition

$$
w=0 \text { on } B .
$$

Under the assumptions that equations (7.1) and (7.13) have unique solutions and the assumptions on the function $f$, and in view of the positivity lemma demonstrated above, we see that $u$ may be represented in the form

$$
u=\operatorname{Max} w[x, t ; v] \text {. }
$$

This leads to

Theorem 7.1. If $u$ is the unique solution of equation (7.1) and is zero on $B$ and $w$ is the unique solution of equations (7.13) and (7.14) for each $v$, then $u$ may be represented in the form given in equation (7.15).

Remark. The customary remarks concerning lower bounds and representation in terms of a maximization over a restricted class of functions $v$ containing $u$ apply.

Newtonian Sequence. We introduce, as before, the Newtonian approximation sequence $\left\{u_{n}\right\}$ specified by

$$
\begin{gathered}
\left(u_{0}\right)_{t}-\left(\dot{u}_{0}\right)_{x x}-f^{\prime}\left(v_{0}\right) u_{0}=f\left(v_{0}\right)-v_{0} f^{\prime}\left(v_{0}\right) ; u_{0}=0, \quad(x, t) \text { on } B \\
\left(u_{n+1}\right)_{t}-\left(u_{n+1}\right)_{x x}-f^{\prime}\left(u_{n}\right) u_{n+1}=f\left(u_{n}\right)-u_{n} f^{\prime}\left(u_{n}\right) \\
u_{n+1}=0, \quad(x, t) \quad \text { on } B, \quad n=0,1,2, \cdots .
\end{gathered}
$$

For convenience we use the notation introduced earlier,

$$
H[u] \equiv u_{t}-u_{x x}
$$

so that equation (7.16) may be rewritten as

$$
\begin{aligned}
& H\left[u_{0}\right]-f^{\prime}\left(v_{0}\right) u_{0}=f\left(v_{0}\right)-v_{0} f^{\prime}\left(v_{0}\right), \quad u_{0}=0 \text { on } B, \\
& H\left[u_{n+1}\right]-f^{\prime}\left(u_{n}\right) u_{n+1}=f\left(u_{n}\right)-u_{n} f^{\prime}\left(u_{n}\right), \\
& u_{n+1}=0 \text { on } B, \quad n=0,1,2, \cdots .
\end{aligned}
$$


It is known (see DoETsch, [16], p. 358) that the solution of the problem

$$
u_{t}-u_{x x}=f(x, t), \quad(x, t) \varepsilon R,
$$

where $f(x, t)$ is continuous on the rectangle $R$ and

$$
u=0, \quad(x, t) \varepsilon B,
$$

may be represented in the form

$$
u=\int_{0}^{1} d y \int_{0}^{t} K(x, y ; t-s) f(y, s) d s .
$$

The kernel $K$ is given by

$$
K(x, y ; t)=\frac{1}{2}\left[\Theta_{3}\left(\frac{x-y}{2}, t\right)-\Theta_{3}\left(\frac{x+y}{2}, t\right)\right],
$$

where the theta function is defined, following DoETsch, to be

$$
\Theta_{3}(v, t)=1+2 \sum_{k=1}^{\infty} e^{-k^{2} \pi^{2} t} \cos 2 k \pi v .
$$

A direct consequence of the positivity lemma and the representation in equation (7.21) is the fact that

$$
K(x, y ; t) \geqq 0 ; \quad 0 \leqq x, y \leqq 1, \quad 0 \leqq t \leqq T
$$

(cf. the proof of the lemma in Section 4).

It follows that $u_{n+1}$ satisfies the integral equation

$$
\begin{aligned}
& u_{n+1}=\int_{0}^{1} d y \int_{0}^{t} K(x, y ; t-s)\left[f\left(u_{n}\right)+f^{\prime}\left(u_{n}\right)\left(u_{n+1}-u_{n}\right)\right] d s, \\
& n=0,1,2, \cdots .
\end{aligned}
$$

Once again defining the norm of $u_{n+1}$ in the domain $R$ by the equation

$$
\left\|u_{n+1}\right\|=\operatorname{Max}_{(x, t) \ell R}\left|u_{n+1}(x, t)\right|,
$$

we find

$$
\left\|u_{n+1}\right\| \leqq \operatorname{Max}_{0 \leq x, y \leq 1} \int_{0}^{T} K(x, y ; T-s)\left[m+m\left(\left\|u_{n+1}\right\|+1\right)\right] d s .
$$

Here we have assumed that

$$
\operatorname{Max}\left\{\left|f\left(u_{n}\right)\right|,\left|f^{\prime}\left(u_{n}\right)\right|\right\} \leqq m<\infty,
$$

provided that $\left|u_{n}\right| \leqq 1$. The existence of the improper integral $\int_{0}^{T} K(x, y ; T-s) d s$ follows from the fact that $K$ may be written in the form

$$
K(x, y ; t)=\frac{1}{\sqrt{4 \pi t}} \sum_{n=-\infty}^{+\infty}\left\{e^{-(x-y+2 n)^{2 / 4 t}}-e^{-(x+y+2 n)^{2 / 4}}\right\}
$$


(see Doetsch, [16], and Courant-Hilbert, [15], V. 2, p. 158). If we put

$$
\underset{0 \leqq x, y \leqq 1}{\operatorname{Max}} \int_{0}^{T} K(x, y ; T-s) d s=k,
$$

then equation (7.27) becomes

$$
\left\|u_{n+1}\right\| \leqq \frac{2 k m}{1-k m},
$$

from which we see that

$$
\text { || } u_{n+1} \| \leqq 1
$$

provided that $k m \leqq \frac{1}{3}$. For fixed $m$, this can be achieved by choosing $T$ sufficiently small. Furthermore, if $v_{0}$ and $T$ are chosen so that $\left\|v_{0}\right\| \leqq 1$ and $\mathrm{km} \leqq \frac{1}{3}$, the induction may be started with $u_{0}$. This establishes the existence of a domain $D$ on which the members of the sequence $\left\{u_{n}\right\}$ are uniformly bounded.

Under our usual assumptions concerning strict convexity of $f(u)$ and uniform boundedness of $f(u)$ and its first two derivatives, it follows that the sequence $\left\{u_{n}\right\}$ converges monotonically and quadratically to a limit function $U$ in the domain $D$. In addition, the convergence is uniform throughout the domain $D$. If now in formula (7.25) we pass to the limit by allowing $n$ to become infinite, we find that $U$ satisfies the equation

$$
U=\int_{0}^{1} d y \int_{0}^{t} K(x, y ; t-s) f(U(y, s)) d s,
$$

from which it follows that $U$ is the solution of equation (7.1). The passage to the limit is justified by remembering that $K$, though unbounded, behaves like const./ $\sqrt{t-s}$ for $s$ in the neighborhood of $t$, which is integrable, and the expression in brackets in equation (7.25) converges uniformly.

\section{Abstract Formulation and Discussion}

Having now examined a number of nonlinear ordinary and partial differential equations which can be linearized through use of the maximum operation, let us outline the fundamental notions in more abstract fashion. Then, in the discussion of Section 9, we shall indicate extensions of the basic idea to other classes of nonlinear problems, including systems of differential equations, integral equations, the calculus of variations, etc.

8. The Equation $L[u]=f(u, x)$. We consider the problem of determining a function $u=u(x)$, where $x$ is an element of the $n$-dimensional Euclidean space $R^{n}$, which satisfies an equation of the form

$$
L[u]=f(u, x) .
$$


Here we assume that $x$ lies in the domain $D$, the boundary of which is $B$. The operator $L$ is a linear operator which we take to be an ordinary or partial differential operator possessing certain additional properties, such as positivity, to be made more precise below, and $f(u, x)$ is a strictly convex function of $u$ for all $x$ in $D$, is continuous in $u$ and $x$, and has a bounded second partial derivative with respect to $u$ for all $u$ and $x$ which enter the discussion.

In addition, it is assumed that appropriate boundary conditions on $u$ are given so that equation (8.1), with the assigned boundary conditions, has a unique solution in the domain $D$. We take this condition to be of the form

$$
u=0, \quad x \varepsilon B,
$$

for if the condition $u=\bar{u}$ on $B$ should be assigned and if $u_{1}$ is a function satisfying $L\left[u_{1}\right]=0$ and $u_{1}=\bar{u}$ on $B$, then the difference function $u_{2}=u-u_{1}$ is zero on the boundary and satisfies the equation

$$
L\left[u_{2}\right]=L[u]=f(u, x)=f\left(u_{1}+u_{2}, x\right)=g\left(u_{2}, x\right)
$$

where $g_{2}$ is also convex in $u_{2}$.

We shall not, in what follows, specifically indicate the dependence of the function $f$ on $x$, and we shall denote partial derivatives of $f$ with respect to $u$ by means of primes.

Representation Theorem. In view of the uniform convexity of $f(u)$ in the domain $D$, equation (8.1) may be written in the form

$$
L[u]=\operatorname{Max}_{v}\left[f(v)+(u-v) f^{\prime}(v)\right]=f(v)+(u-v) f^{\prime}(v)+p,
$$

where $p$ is a non-negative function defined on $D$ for any particular choice of $v$. The maximization is over all functions $v$ defined on $D$. Since we know that the maximum is actually attained for $v=u$, we may suitably restrict the class of admissible functions $v$ to some class including $u$, as has been remarked in earlier sections. In particular, $v$ is to be sufficiently smooth so that a unique solution of the associated linear equation defined below is obtained for each choice of an admissible function $v$.

We now introduce the associated linear equation for the function $w=$ $w[x ; v]$,

$$
L[w]=f(v)+(w-v) f^{\prime}(v),
$$

which, with the boundary condition $w=0$ on $B$, we assume uniquely determines $w$ for each admissible choice of the function $v$. In particular, if $v=u$, then the solution becomes

$$
w=w[x ; u]=u .
$$

We next make the following positivity assumption concerning $L$ : If $z$, which is zero on $B$, satisfies the operator inequality

$$
L[z]-f^{\prime}(v) z \geqq 0
$$


throughout the domain $D$, for all admissible functions $v$, then

$$
z \geqq 0
$$

on $D$. In earlier sections we have given examples of operators which do have this property.

The representation for the assumed solution of equation (8.1) now follows immediately. The difference function $u-w$, according to equations (8.4) and (8.5), satisfies the inequality

$$
L[u-w] \geqq f^{\prime}(v)(u-w), \quad x \varepsilon D,
$$

and $u-w$ is zero on the boundary. From the positivity assumption it then follows that

$$
u \geqq w[x ; v]
$$

for any admissible choice of $v$. In view of equation (8.6) this implies that the representation

$$
u=\underset{v}{\operatorname{Max}} w[x ; v]
$$

holds.

Let us state this as

Theorem 8.1. If $L$ is a linear operator possessing the positivity property of equations (8.7) and (8.8) and $f(u)$ is a strictly convex function of $u$ for all finite $u$, then $u$, the solution of the nonlinear equation (8.1) and the boundary condition (8.2), which is assumed to exist and be unique, may be represented in the form

$$
u=\operatorname{Max}_{\bullet} w[x ; v]
$$

where $w=w[x ; v]$ is the solution of the associated linear equation (8.4), which is also assumed to exist and be unique for each admissible function $v$.

In many instances, as we have already seen, the requisite existence and uniqueness proofs may already be available. The establishment of the positivity property may, accordingly, be expected to constitute the major obstacle in practical applications. In some problems, as in Sections 4,6 , and 7 , the positivity property is equivalent to the fact that certain kernel (Green's) functions are of one sign in the region $D$. Other positivity results are referred to in the discussion of Section 9.

Immediate consequences of the representation theorem are lower bounds for $u$ in the form of the function $w=w\left[x ; v_{1}\right]$, for any choice of $v_{1}$; the fact that $u$ satisfies the (nonlinear integral) equation $u=w[x ; u]$; and, in the event that $f$ is a random function of $x$ and $u$, a representation for the distribution function of $u$, similar to that obtained for the stochastic Riccati equation in Section 1.

Finally, let us show how to construct a sequence of functions that converge monotonically to $u$; that is, we produce a convergent minimizing sequence for the functional $w[x ; v]$. 
Newtonian Approximation. Let an initial approximation $v_{0}(x)$ be selected and determine the function $u_{0}$ as the solution of the linear inhomogeneous equation

$$
L\left[u_{0}\right]=f\left(v_{0}\right)+\left(u_{0}-v_{0}\right) f^{\prime}\left(v_{0}\right), \quad u=0, \quad x \varepsilon B .
$$

Then determine an improved admissible function $v_{1}$ as the function which maximizes the expression $f(v)+\left(u_{0}-v\right) f^{\prime}(v)$, point by point throughout the region $D$. In view of the convexity of $f$, this function is $v=v_{1}=u_{0}$. The function $u_{1}$ is then determined as the solution of the equation

$$
L\left[u_{1}\right]=f\left(u_{0}\right)+\left(u_{1}-u_{0}\right) f^{\prime}\left(u_{0}\right), \quad u_{1}=0, \quad x \varepsilon B .
$$

An improved admissible function $v=v_{2}$ is then determined as the function which maximizes the expression $f(v)+\left(u_{1}-v\right) f^{\prime}(v)$; this function is $v=v_{2}=u_{1}$. Continuing in this fashion we are led to the sequence of functions $\left\{u_{n}\right\}$ defined by the equations

$$
\begin{aligned}
L\left[u_{0}\right] & =f\left(v_{0}\right)+\left(u_{0}-v_{0}\right) f^{\prime}\left(v_{0}\right), \\
L\left[u_{n+1}\right] & =f\left(u_{n}\right)+\left(u_{n+1}-u_{n}\right) f^{\prime}\left(u_{n}\right), \\
u_{k}=0, \quad x \varepsilon B, \quad k=0,1,2, \cdots . &
\end{aligned}
$$

Let us first consider the uniform boundedness of the sequence $\left\{u_{n}\right\}$. We assume that the operator $L$ has a left inverse, which we denote by $T$, so that we have

$$
\begin{aligned}
u_{0} & =T\left[f\left(v_{0}\right)+\left(u_{0}-v_{0}\right) f^{\prime}\left(v_{0}\right)\right], \\
u_{n+1} & =T\left[f\left(u_{n}\right)+\left(u_{n+1}-u_{n}\right) f^{\prime}\left(u_{n}\right)\right] .
\end{aligned}
$$

Then we assume that $T$ is a bounded additive operator for which

$$
\|T f\| \leqq k\|f\|
$$

for some $k<\infty$. Here $f$ and $T f$ need not be in the same space so that the two norms need not necessarily be the same. In the previous sections we have defined the norm of the continuous function $v$ on the domain $D$ to be

$$
\|v\|=\operatorname{Max}_{x \varepsilon D}|v| \text {. }
$$

In addition, we assume that if $|u| \leqq 1$, then

$$
\infty>m>\operatorname{Max}\left\{|f(u)|,\left|f^{\prime}(u)\right|, \frac{1}{2}\left|f^{\prime \prime}(u)\right|\right\} .
$$

We now proceed by induction. If $\left\|u_{n}\right\| \leqq 1$, then

$$
\left\|u_{n+1}\right\| \leqq k m\left(\left\|u_{n+1}\right\|+2\right),
$$

so that

$$
\left\|u_{n+1}\right\| \leqq \frac{2 k m}{1-k m}
$$


which is less than unity, provided that $k m<\frac{1}{3}$. Furthermore, if the initial approximation $v_{0}$ is chosen so that

$$
\left\|v_{0}\right\| \leqq \frac{2 k m}{1-k m}<1
$$

then we shall be able to begin the induction for $n=0$. This establishes the uniform boundedness of the sequence $\left\{u_{n}\right\}$ on the domain $D$ under the assumption that $\mathrm{km}<\frac{1}{3}$.

Let us now see that the sequence $\left\{u_{n}\right\}$ is actually monotone. From equation (8.15) we see that

$$
\begin{aligned}
L\left[u_{n+1}\right] & =f\left(u_{n}\right)+\left(u_{n+1}-u_{n}\right) f^{\prime}\left(u_{n}\right), \\
L\left[u_{n}\right] & =f\left(u_{n-1}\right)+\left(u_{n}-u_{n-1}\right) f^{\prime}\left(u_{n-1}\right),
\end{aligned}
$$

where

$$
u_{n}=u_{n+1}=0 \quad x \varepsilon B, \quad n=0,1,2, \cdots .
$$

The convexity of $f(u)$ which was assumed above implies that

$$
L\left[u_{n}\right] \leqq f\left(u_{n}\right)+\left(u_{n}-u_{n}\right) f^{\prime}\left(u_{n}\right),
$$

from which it follows that

$$
L\left[u_{n+1}-u_{n}\right] \geqq\left(u_{n+1}-u_{n}\right) f^{\prime}\left(u_{n}\right), \quad u_{n+1}-u_{n}=0, \quad x \varepsilon B .
$$

The positivity property of $L$ then yields the result that

$$
u_{n+1}-u_{n} \geqq 0, \quad n=0,1,2, \cdots,
$$

which completes the proof of the monotone character of the sequence $\left\{u_{n}\right\}$.

Since the sequence $\left\{u_{n}\right\}$ is uniformly bounded from above (for $k m<\frac{1}{3}$ ) and monotone increasing in the domain $D$, the sequence converges to a limit function $U(x)$. If now we make the assumption that

$$
\lim _{n \rightarrow \infty} T\left[f u_{n}\right]=T[f(u)]
$$

and further assume that $L$ is the left inverse of $T$,

$$
L T=I,
$$

then from equation (8.15) we find

$$
L[U]=f(U), \quad U=0, \quad x \varepsilon B ;
$$

that is, the limit function $U$ is the (assumed unique) solution of equations (8.1) and (8.2).

Let us now summarize these observations in the form of

Theorem 8.2. The sequence $\left\{u_{n}\right\}$ defined by equation (8.15) converges monotonically to the solution of equations (8.1) and (8.2) under the assumptions 
(a) L has the positivity property of equations (8.7) and (8.8);

(b) the left inverse of $L, T$, is additive and bounded with $\|T u\| \leqq k\|u\|, k<\infty$;

(c) $k m<\frac{1}{3}$, where $m \geqq \operatorname{Max}\left\{|f(u)|,\left|f^{\prime}(u)\right|, \frac{1}{2}\left|f^{\prime \prime}(u)\right|\right\}$ for $|u| \leqq 1$;

(d) $L T=I$.

We now consider the rate of convergence of the sequence $\left\{u_{n}\right\}$; in particular, we show that the sequence converges quadratically, which is typical of a Newtonian approximation scheme. From equations (8.1) and (8.15) we find

$$
L\left[u-u_{n}\right]=f(u)-f\left(u_{n-1}\right)-\left(u_{n}-u_{n-1}\right) f^{\prime}\left(u_{n-1}\right) .
$$

This equation may be rewritten

$$
L\left[u-u_{n}\right]=f(u)-f\left(u_{n-1}\right)-\left(u-u_{n-1}\right) f^{\prime}\left(u_{n-1}\right)-\left(u_{n}-u\right) f^{\prime}\left(u_{n-1}\right) .
$$

Making use of the assumption $T L=I$ we find

$$
u-u_{n}=T\left[f\left(u_{n}\right)-f\left(u_{n-1}\right)-\left(u-u_{n-1}\right) f^{\prime}\left(u_{n-1}\right)+\left(u-u_{n}\right) f^{\prime}\left(u_{n-1}\right)\right] .
$$

Consequently, using the mean value theorem and the properties of the operator $T$, we have

$$
\left\|u-u_{n}\right\| \leqq k\left\{m\left\|u-u_{n-1}\right\|^{2}+m\left\|u-u_{n}\right\|\right\}
$$

so that

$$
\left\|u-u_{n}\right\| \leqq \frac{k m}{1-k m}\left\|u-u_{n-1}\right\|^{2}, \quad n=1,2, \cdots .
$$

In addition, in so far as numerical computation is concerned, it is desirable to have an estimate available for the size of the changes to be expected from iteration to iteration. It is easily shown, as above, that

$$
\left\|u_{n+1}-u_{n}\right\| \leqq \frac{k m}{1-k m}\left\|u-u_{n-1}\right\|^{2}, \quad n-1,2, \cdots .
$$

In addition, since $\mathrm{km}<\frac{1}{3}$, this argument shows that the sequence $\left\{u_{n}\right\}$ converges uniformly on the domain $D$ to the limit function $U(x)$.

9. Discussion. In previous sections we have discussed at some length how various nonlinear ordinary and partial differential equations may be linearized through use of the maximum or minimum operation. We now wish to sketch briefly a number of extensions of this technique to variational problems, first order quasilinear partial differential equations, systems of differential equations, and to equations in which the Min-Max operation can be utilized.

Finally we shall mention a number of related positivity results established by Chaplygin, [3], Wilkins, [48], Pólya, [37], and others.

Calculus of Variations. BeLlman, [7], has shown that the problem of maximizing the integral 


$$
J[y]=\int_{0}^{T} F(x, y) d t
$$

subject to the conditions

$$
\frac{d x}{d t}=G(x, y), \quad x(0)=c,
$$

leads to the functional equation

$$
u_{T}=\operatorname{Max}_{v}\left[F(c, v)+G(c, v) u_{c}\right], \quad u(c, 0)=0,
$$

under suitable regularity conditions on the functions $F$ and $G$, where the function $u=u(c, T)$ is defined by the equation

$$
u(c, T)=\operatorname{Max}_{y} J[y]
$$

for $T \geqq 0,-\infty<c<+\infty$. We observe that equation (9.3) is already in a form which is suitable for treatment by our basic technique. This equation, then, provides an example of the natural occurrence of equations of this type.

We introduce the associated linear equation

$$
w_{T}=F(c, v)+G(c, v) w_{c}, \quad w(c, 0)=0,
$$

and observe that the difference function $z=u-w$ satisfies the inequality

$$
z_{T}-G z_{c} \geqq 0, \quad z(c, 0)=0,
$$

from which the non-negativity of $z$ for $T \geqq 0$ follows immediately. This leads to the representation

$$
u=\operatorname{Max} w[c, T ; v], \quad T \geqq 0 .
$$

Quasilinear First Order Equations. Let us consider the conservation law

$$
u_{t}+[f(u)]_{x}=0, \quad u(x, 0)=g(x), \quad T \geqq t \geqq 0,
$$

where we assume that $f(u)$ is strictly convex and has a finite second derivative for finite $u$. The case $f(u)=\frac{1}{2} u^{2}$ is a limiting case of the Bateman-Burger equation

$$
u_{t}+u u_{x}=\mu u_{x x},
$$

which occurs in mathematical models of the theory of turbulence (see HopF, [20], and CoLE, [14]). The situation when the initial function $g(x)$ is assumed bounded and measurable has been extensively investigated by LAx, [26, 27, 28], and OLEİNIK, [31, 32], and has led to the creation of the theory of weak solutions of equation (9.8).

Equation (9.8) may be rewritten as

$$
u_{t}+f^{\prime}(u) u_{x}=0
$$


We introduce a function $U=U(x, t)$ for which

$$
U_{x}=u,
$$

so that equation (9.10) becomes

$$
U_{x t}+f^{\prime}\left(U_{x}\right) U_{x x}=0
$$

or upon integration with respect to $x$

$$
U_{t}+f\left(U_{x}\right)=0
$$

an equation which holds modulo a function of $t$. By adding a suitable function of $t$ to $U$, if necessary, we may make the strict equality hold. Furthermore, by adding a suitable constant to $U$ we may arrange to have $U(0,0)=0$, so that $U$ satisfies the initial condition

$$
U(x, 0)=\int_{0}^{x} g(s) d s=G(x) .
$$

The associated linear equation is

$$
w_{t}+f(v)+\left(w_{x}-v\right) f^{\prime}(v)=0, \quad w(x, 0)=G(x),
$$

and the difference function $z=U-w$ satisfies the equation

$$
z_{t}+z_{x} f^{\prime}(v)=-p, \quad z(x, 0)=0,
$$

where $p$ is non-negative for each choice of $v$. It follows that

$$
z=U-w \leqq 0, \quad t \geqq 0 .
$$

Furthermore, it is readily seen that $w=U$ if $v=U_{x}=u$. Thus we have the representation for the solution of equation (9.13)

$$
U=\operatorname{Min}_{v} w[x, t ; v],
$$

and the minimizing function is $v=u$, where $u$ is a solution of equation (9.8).

Professor P. D. LAx has pointed out that the representation of equation (9.18) is equivalent to his own representation formula, [28, p. 45], which in turn generalizes a formula of HopF, [20]. To see this we observe that the minimizing function $u$ is constant along characteristics and therefore that it is sufficient to admit those functions $v$ which are constant along characteristics. Furthermore, the value of the solution $u$ of equation (9.15) at a point $P$ depends only on the values of $v$ along the characteristics through $P$. Therefore it is sufficient to consider functions $v$ which are constant. For constant $v$ equation (9.15) can be solved explicitly, which leads to the desired formula.

The representation of equation (9.18) leads us, as in earlier sections, to consider the Newtonian approximating sequence $\left\{U_{n}\right\}$, defined by the relations

$$
\begin{gathered}
\left(U_{0}\right)_{t}+f\left(v_{0}\right)+\left(\left(U_{0}\right)_{x}-v_{0}\right) f^{\prime}\left(v_{0}\right)=0, \\
\left(U_{n+1}\right)_{t}+f\left(\left(U_{n}\right)_{x}\right)+\left(\left(U_{n+1}\right)_{x}-\left(U_{n}\right)_{x}\right) f^{\prime}\left(\left(U_{n}\right)_{x}\right)=0, \\
U_{k}(x, 0)=G(x), \quad k=0,1,2, \cdots .
\end{gathered}
$$


LAx has also observed that the sequence $\left\{U_{n}\right\}$ converges to $U(x)$.

Now consider the equation

$$
u_{t}+[f(u)]_{x}=\mu u_{x x}, \quad u(x, 0)=g(x), \quad \mu>0,
$$

which, as before, upon the introduction of the function $U$, where $U_{x}=u$, becomes

$$
U_{t}+f\left(U_{x}\right)=\mu U_{x x}, \quad U(x, 0)=G(x),
$$

or

$$
U_{t}+\operatorname{Max}_{v}\left[f(v)+\left(U_{x}-v\right) f^{\prime}(v)\right]=\mu U_{x x} .
$$

We let $w$ be the solution of the linear equation

$$
w_{t}+f(v)+f^{\prime}(v)\left(w_{x}-v\right)=\mu w_{x x}, \quad w(x, 0)=G(x) .
$$

Then it may be shown that

$$
U=\operatorname{Inf}_{v} w[x, t ; v]
$$

An interesting question to ask is whether or not the limiting process of taking the infimum over a class of admissible $v$ can be interchanged with that of letting $\mu$ tend to zero so that the equation

$$
\lim _{\mu \rightarrow 0} \operatorname{Inf}_{\bullet} w=\operatorname{Inf} \lim _{\nu \rightarrow 0} w
$$

holds.

Systems of Equations. Some of the foregoing theory may be carried over to considerations concerning systems of equations. Let us, for example, consider the nonlinear system of ordinary first order differential equations

$$
u^{\prime}(x)=f(u), \quad u(0)=c,
$$

where $u, f$, and $c$ are all $n$-dimensional vectors. In addition, let us suppose that we may represent $f(u)$ in the form

$$
f(u)=\operatorname{Max}_{v}[f(v)+J(v)(u-v)]
$$

where $J$, the Jacobi matrix, is defined by

$$
J(v)=\left[\begin{array}{llll}
\frac{\partial f_{1}}{\partial v_{1}} & \frac{\partial f_{1}}{\partial v_{2}} & \cdots & \frac{\partial f_{1}}{\partial v_{n}} \\
\frac{\partial f_{n}}{\partial v_{1}} & \frac{\partial f_{n}}{\partial v_{2}} & \cdots & \frac{\partial f_{n}}{\partial v_{n}}
\end{array}\right],
$$

and the maximization is over all vector functions $v$. This will be the case if each of the components of $f(u)$ is a strictly convex function of the vector $u$ (see Section 2). The requisite positivity property is that if 


$$
z^{\prime}-J(v) z=p \geqq 0, \quad z(0)=0,
$$

then

$$
z \geqq 0, \quad x \geqq 0 .
$$

As is customary, we consider vectors and matrices to be non-negative if their components are.

It is not unrestrictedly true that the positivity property holds. A sufficient condition, though, is that the off-diagonal terms of $J$ be non-negative, [13]. By making the change of dependent variable

$$
y_{i}=z_{i} \exp \left\{-\int_{0}^{x} \frac{\partial f_{i}}{\partial v_{i}} d s\right\},
$$

equation (9.29) becomes

$$
y_{i}^{\prime}=\sum_{i \neq j} \frac{\partial f_{i}}{\partial v_{i}} y_{i}+p_{i} \exp \left\{-\int_{0}^{x} \frac{\partial f_{i}}{\partial v_{i}} d s\right\}, \quad y_{j}(0)=0,
$$

from which the assertion follows. In the event that $J$ is a constant matrix, the above condition is also necessary.

Min-Max Operation. Some nonlinear equations may be linearized by use of the Min-Max operation. As an illustration of this remark consider the equation

$$
u^{\prime \prime}=f\left(u^{\prime}, u\right), \quad u(0)=u(b)=0,
$$

where we assume that $b$ is sufficiently small so as to guarantee the existence of a unique solution $u=u(x), 0 \leqq x \leqq b$. Let us assume that the sufficiently smooth function $f\left(u^{\prime}, u\right)$ may be represented in the form

$$
\begin{aligned}
f\left(u^{\prime}, u\right)=\operatorname{Max}_{v_{1}} \operatorname{Min}_{v} & {\left[f\left(v_{1}, v\right)+\left(u^{\prime}-v_{1}\right) f_{v_{1}}+(u-v) f_{v}\right] } \\
& =\operatorname{Min}_{v} \operatorname{Max}_{v_{1}}\left[f\left(v_{1}, v\right)+\left(u^{\prime}-v_{1}\right) f_{v_{1}}+(u-v) f_{v}\right],
\end{aligned}
$$

where the maximization is over all functions $v_{1}$ defined on the interval $[0, b]$, and the minimization is over all functions $v$ defined on that interval. The interchange of the operators is assumed allowable. If $f$ is strictly convex in $u^{\prime}$ and strictly concave in $u$, the representations in equation (9.34) hold. The saddle point occurs at $v_{1}=u^{\prime}$ and $v=u$.

To obtain a representation for the solution of equation (9.33) in terms of the Min-Max operation applied to the solutions of an associated linear equation we may proceed in the following manner. Place $v=u$. Then

$$
\begin{gathered}
u^{\prime \prime}=\operatorname{Max}_{\mathbf{\prime}_{1}}\left[f\left(v_{1}, u\right)+\left(u^{\prime}-v_{1}\right) f_{v_{1}}\right], \\
u^{\prime \prime} \geqq f\left(v_{1}, u\right)+\left(u^{\prime}-v_{1}\right) f_{v_{1}} .
\end{gathered}
$$

Next introduce $w=w\left[x ; v_{1}, v\right]$ as the solution-assumed to exist for admissible 
classes of functions $v_{1}$ and $v$-of the equation

$$
w^{\prime \prime}=f\left(v_{1}, v\right)+\left(w^{\prime}-v_{1}\right) f_{v_{1}}+(w-v) f_{v}, \quad w(0)=w(b)=0 .
$$

When $v=u$ equation (9.37) reduces to

$$
w^{\prime \prime}=f\left(v_{1}, u\right)+\left(w^{\prime}-v_{1}\right) f_{v_{1}}, \quad w(0)=w(b)=0 .
$$

Using the positivity results of Sections 4 and 5 we see that for $b$ sufficiently small

$$
z=u-w\left[x ; v_{1}, u\right] \leqq 0, \quad 0 \leqq x \leqq b .
$$

This in turn implies that

$$
u=\operatorname{Min}_{v_{1}} w\left[x ; v_{1}, u\right]
$$

and lastly that

$$
u(x) \leqq \operatorname{Max}_{v} \operatorname{Min}_{v_{1}} w\left[x ; v_{1}, v\right], \quad 0 \leqq x \leqq b .
$$

Now let us place $v_{1}=u^{\prime}$, which yields

$$
u^{\prime \prime}=\operatorname{Min}\left[f\left(u^{\prime}, v\right)+(u-v) f_{v}\right]
$$

or

$$
u^{\prime \prime} \leqq f\left(u^{\prime}, v\right)+(u-v) f_{v} .
$$

The function $w\left[x ; u^{\prime}, v\right]$ satisfies the equation

$$
w^{\prime \prime}=f\left(u^{\prime}, v\right)+(w-v) f_{v}, \quad w(0)=w(b)=0 .
$$

The difference function $u(x)-w\left[x ; u^{\prime}, v\right]=z$ satisfies the differential inequality

$$
z^{\prime \prime} \leqq z f_{v}, \quad z(0)=z(b)=0,
$$

which implies, according to the lemma of Section 4 , that

$$
z \geqq 0, \quad 0 \leqq x \leqq b,
$$

provided that $b$ is sufficiently small. This inequality implies that

$$
\begin{gathered}
u \geqq w\left[x ; u^{\prime}, v\right], \\
u \geqq \underset{v}{\operatorname{Max}_{v} w\left[x ; u^{\prime}, v\right],} \\
u \geqq \operatorname{Min}_{v_{1}} \operatorname{Max}_{v} w\left[x ; v_{1}, v\right] .
\end{gathered}
$$

From inequalities (9.41) and (9.49) we see that

$$
\operatorname{Min}_{v_{1}} \operatorname{Max}_{v} w\left[x ; v_{1}, v\right] \leqq u \leqq \operatorname{Max}_{v} \operatorname{Min}_{v_{1}} w\left[x ; v_{1}, v\right], \quad 0 \leqq x \leqq b .
$$

But, in general. we have

$$
\underset{v_{1}}{\operatorname{Min}} \operatorname{Max} w\left[x ; v_{1}, v\right] \geqq \underset{v}{\operatorname{Max}} \underset{v_{1}}{\operatorname{Min}} w\left[x ; v_{1}, v\right],
$$


so that

$$
u(x)=\operatorname{Min}_{v_{1}} \operatorname{Max}_{v} w\left[x ; v_{1}, v\right]=\operatorname{Max}_{v} \operatorname{Min}_{v_{1}} w\left[x ; v_{1}, v\right], \quad 0 \leqq x \leqq b,
$$

which is the desired representation on the sufficiently small interval $[0, b]$. Also it establishes that the Min-Max and Max-Min operations are interchangeable when applied to $w\left[x ; v_{1}, v\right]$.

From the representation of equation (9.52) we obtain upper and lower bounds for $u(x)$ on the interval $[0, b]$ in the form

$$
\begin{array}{cc}
\operatorname{Min}_{v_{1}} w\left[x ; v_{1}, \bar{v}\right] \leqq u(x), & \text { for all admissible } \bar{v} \\
u(x) \leqq \operatorname{Max}_{v} w\left[x ; \bar{v}_{1}, v\right], & \text { for all admissible } \bar{v}_{1} .
\end{array}
$$

This representation is valid for more general linear operators $L$, having similar positivity properties, for which unique solutions of the analogues of equations (9.34) and (9.37) exist. Sufficient for this is that if

$$
L[z] \geqq z f_{v}+z^{\prime} f_{v_{1}}, \quad z=0 \quad \text { on the boundary } B,
$$

then

$$
z \leqq 0 \quad \text { on the domain } D \text {. }
$$

The representation in equation (9.52) leads, of course, to various successive approach schemes for the solution of equation (9.33).

An example of a different type, arising from the study of multi-stage games, occurs in SHAPLEY, [41].

Nonlinear Integral Equations. Let us consider, as a concrete example, the nonlinear integral equation

$$
u(x)=g(x)+\lambda \int_{0}^{1} K(x, y) u^{2}(y) d y, \quad K \geqq 0,
$$

where $\lambda$ is a sufficiently small positive parameter. This equation may be rewritten in the form

$$
u(x)=g(x)+\lambda \operatorname{Max}_{v} \int_{0}^{1} K(x, y)\left[2 v(y) u(y)-v^{2}(y)\right] d y
$$

where the maximum is attained for $v=u$. The associated linear equation is

$$
w=g+\lambda \int_{0}^{1} K(x, y)\left[2 v w-v^{2}\right] d y
$$

so that the difference equation for $z=u-w$ becomes

$$
z=2 \lambda \int_{0}^{1} K(x, y) v(y) z(y) d y+p
$$

where $p$ is a non-negative function for each choice of the function $v$. 
If now we assume that $g(x) \geqq \epsilon>0$ on the interval $[0,1]$, then from equation (9.56) we see that $u(x)$ will be positive, and we may also assume that all admissible functions $v(x)$ are also positive. Furthermore, if $K(x, y)$ has the form

$$
K(x, y)=\left[\begin{array}{ll}
h(y), & 0 \leqq y \leqq x, \\
0, & 1 \geqq y>x,
\end{array}\right.
$$

it is clear that $z(x)$ will be non-negative on some right hand neighborhood of the origin. This establishes the requisite positivity property for some sufficiently small interval $0 \leqq x \leqq b$.

Positivity Results. Scattered throughout the literature are various positivity results which may be utilized in studies similar to this one. Let us briefly call attention to several.

Consider the initial value problem

$$
u^{\prime \prime}=f(u), \quad u(0)=u_{0}, \quad u^{\prime}(0)=u_{1}, \quad 0 \leqq x,
$$

where $f(u)$ is a convex function with a finite second derivative for finite $u$. Here the positivity property amounts to the requirement that

$$
z^{\prime \prime}-f^{\prime}(v) z \geqq 0, \quad z(0)=z^{\prime}(0)=0,
$$

is to imply

$$
z \geqq 0
$$

in some interval $[0, b]$. It is clear that the inequality (9.63) holds in some right hand neighborhood of $x=0$, but how large is this interval? The answer is provided in works of Chapygin, Petrov, [33], Wilkins, [48], and Babkin, [3], where it is shown that $z$ is non-negative in the interval $[0, b]$, provided that the equation

$$
z^{\prime \prime}-f^{\prime}(v) z=0
$$

(the classical Jacobi condition) has a solution which does not vanish when $0<x<b$.

Other interesting results are due to Pólya, [37], and Poincaré, [36].

Westrhal, [39], and Prodi, [46], have derived certain positivity results concerning the nonlinear heat equation

$$
u_{t}=f\left(u_{x x}, u_{x}, u, x, t\right)
$$

Their results were stimulated by the original result of HAAR, [19].

BeLlman has employed the following lemma: Let $u, v \geqq 0, c>0$ and $u$ satisfy the inequality

then

$$
u \leqq c+\int_{0}^{x} u v d y, \quad x \geqq 0
$$

$$
u \leqq c \exp \left\{\int_{0}^{x} v(y) d y\right\} .
$$

This has been extended to two dimensions by WENDRoFf, [45]. 


\section{BIBLIOGRAPHY}

[1] Aronszajn, N. \& K. Smith, A Characterization of Positive Reproducing Kernels. Applications to Gieen's Functions, University of Kansas, Technical Report 15, 1956.

[2] Aronszajn, N. \& K. Smith, Characterization of Positive Reproducing Kernels. Applications to Green's Functions, American Journal of Mathematics, 79 (1957), pp. 611-622.

[3] Baвkin, B. N., On a Generalization of a Theorem of Academician S.A. Caplygin on a Differential Inequality, Molotov Gos. Univ. Uč. Zap., 8 (1953), pp. 3-6.

[4] Bailex, V: A., Reflection of Waves by an Inhomogeneous Medium, The Physical Review, Ser. 2, 96 (1954), pp. 865-868.

[5] Bellman, R., On the Existence and Boundedness of Solutions of Nonlinear Partial Differential Equations of Parabolic Type, Transactions of the American Mathematical Society, 64 (1948), pp. 21-44.

[6] Bellman, R., The Theory of Dynamic Programming, Bulletin of the American Mathematical Society, 60 (1954), pp. 503-515.

[7] Bellman, R., Dynamic Programming and a New Formalism in the Calculus of Variations-I, Rivista Mat. Univ. Parma, 6 (1955), pp. 193-213.

[8] Bellman, R., On the Non-Negativity of Green's Functions, The RAND Corporation, Paper P-1065, April, 1957.

[9] Bellman, R., Functional Equations in the Theory of Dynamic Programming. II. Nonlinear Differential Equations, Proc. Nat. Acad. Sci. USA., 41 (1955), pp. 482-485.

[10] Bellman, R., Functional Equations in the Theory of Dynamic Programming. V. Positivity and Quasilinearity, Proc. Nat. Acad. Sci. USA, 41 (1955), pp. 743-746.

[11] Bellman, R., On the Representation of the Solution of a Class of Stochastic Differential Equations, Proc. Amer. Math. Soc., 9 (1958), pp. 326-327.

[12] Bellman, R., A Note on Monotone Convergence to Solutions of First Order Differential Equations, Proc. Amer. Math. Soc., to appear.

[13] Bellman, R., I. Glicksberg, \& O. Gross, On Some Variational Problems Occurring in the Theory of Dynamic Programming, Rendiconti Cir. Mat. Palermo, Serie II, 3 (1954), pp. 1-35.

[14] CoLE, J., On a Quasi-linear Parabolic Equation Occurring in Aerodynamics, Quarterly of Applied Mathematics, 9 (1951), pp. 225-236.

[15] Courant, R. \& D. Hilbert, Methoden der Mathematischem Physik, Vols. I and II, Springer, Berlin, 1937.

[16] Doetsch, G., Theorie und Anwendung der Laplace-Transformation, Dover Publications, New York, 1943.

[17] Feller, W., The General Diffusion Operator and Positivity Preserving Semi-Groups in One Dimension, Annals of Mathematics, 60 (1954), pp. 417-446.

[18] Friedrichs, K. O. \& J. J. Stoker, Forced Vibrations of Systems with Nonlinear Restoring Force, Quarterly of Applied Mathematics, 1 (1942), pp. 97-115.

[19] HaAR, A., Über Eindeutigkeit und Analyzität der Lösungen partieller Differentialgleichungen, Atti Congresso Intern. Mat., Bologna, 1928, Vol. III, pp. 5-10.

[20] Hopf, E., The Partial Differential Equation $u_{t}+u u_{x}=\mu u_{x x}$, Communications on Pure and Applied Mathematics, 3 (1950), pp. 201-230.

[21] Kantonovič, L. V., On Newton's Method, Trudy. Mat. Inst. Steklov, 28 (1949), pp. 104144.

[22] Krller, E. G., Some Present Nonlinear Problems of the Electrical and Aeronautical Industries, Quarterly of Applied Mathematics, 2 (1944), pp. 72-86.

[23] Keller, J., On Solutions of $\Delta u=f(u)$, Research Report No. MME-1, New York University, Institute of Mathematical Sciences, 1957.

[24] Keller, J., Electrohydrodynamics I: The Equilibrium of a Charged Gas in a Container, $J$. of Rat. Mech. and Analysis, 5 (1956), pp. 715-724.

[25] Keller, J. B., On Solutions of Nonlinear Wave Equations, New York University, Institute of Mathematical Sciences, Research Report No. MME-3, April, 1957. 
[26] LAx, P., Weak Solutions of Nonlinear Hyperbolic Equations and their Numerical Computation, Communications on Pure and Applied Mathematics, 7 (1954), pp. 159-193.

[27] LAx, P., The Initial Value Problem for Nonlinear Hyperbolic Equations in Two Independent Variables, Chapter 12 of Contributions to the Theory of Partial Differential Equations, Annals of Mathematics Study No. 33, Princeton University Press, Princeton, 1954 .

[28] LAx, P., Initial Value Problems for Nonlinear Hyperbolic Equations, University of Kansas, Technical Report 14, 1955.

[29] Minakshisundaram, S., Fourier Ansatz and Nonlinear Parabolic Equations, Journal of the Indian Mathematical Society, N.S., 7 (1943), pp. 129-143.

[30] Morrey, C. B., Jr., Nonlinear Methods, Chapter 16 of Modern Mathematics for the Engineer, E. F. Beckenbach (ed.), McGraw-Hill Book Company, Inc., New York, 1956.

[31] OlEY̌NIK, O. A., Zadača Koši dlya NelineY̌nh Differencial'nyh Uravnenii Pervovo Poryadka c Razryvnymi Načal'nymi Usloviyami, Trudy Mockovskovo Matematičeskovo Obščestva, 5 (1956), pp. 433-454.

[32] OLEǏnIK, O. A., O Razryvnyh Rešeniyah NelineY̌nyh Differencial'nyh Uravenenii, Doklady Akademii Nauk SSSR, 109 (1956), pp. 1098-1101.

[33] Petrov, V. N., The Limits of Applicability of S. Tchaplygin's Theorem on Differential Inequalities to Linear Equations with Usual Derivatives of the Second Order, Comptes Rendus (Doklady) de L' Academie des Sciences de L'URSS, 51 (1946), pp. 255-258.

[34] Petrovskx, I. G., Lectures on Partial Differential Equations, Interscience Publishers, Inc., New York, 1954.

[35] Picard, E., Mémoire sur la Théorie des Equations aux Dérivées Partielles et la Méthode des Approximations Successives, Journal de Mathématiques (4), 6 (1890), pp. 145-210.

[36] Poincaré, J. H., L'Intermédiaire des Mathématiciens, Vol. I (1894), pp. 141-144.

[37] Pólya, G., On the Mean-value Theorem Corresponding to a Given Linear Homogeneous Differential Equation, Transactions of the American Mathematical Society, 24 (1922), pp. 312-324.

[38] PóLYA, G. \& G. Szegö, Isoperimetric Inequalities in Mathematical Physics, Annals of Mathematics Study No. 27, Princeton University Press, Princeton, 1951.

[39] Prodr, G., Questioni di Stabilitá per Equazioni Nonlineari alle Derivate Parziali di Tipo Parabolico, Atti Accad. Nazl. Lincei Ren., Classe sic. fis. mat. e nat., 10 (1951), pp. 365-370.

[40] Schelkonoff, S., Solution of Linear and Slightly Nonlinear Differential Equations, Quarterly of Applied Mathematics, 3 (1945), pp. 348-355.

[41] Shapley, L., Stochastic Games, Proc. Nat. Acad. Sci. USA, 39 (1953), pp. 1095-1100.

[42] Srdprer, R., Boundary Problems in Non-linear Parabolic Equations, The Journal of the Indian Mathematical Society, N.S., 1 (1934-1935), pp. 125-128.

[43] SтокеR, J. J., Nonlinear Vibrations in Mechanical and Electrical Systems, Interscience Publishers, Inc., New York, 1950.

[44] von Kármán, T., The Engineer Grapples with Nonlinear Problems, Bulletin of the American Mathematical Society, 46 (1940), pp. 615-683.

[45] Wendroff, B., An Integral Inequality, Bull. Amer. Math. Soc., 63 (1957), p. 243.

[46] Westphal, H., Zur Abschätzung der Lösungen Nichtlinearer Parabolischer Differentialgleichungen, Math. Z., 51 (1949), pp. 690-695.

[47] Weyl, H., On the Differential Equations of the Simplest Boundary-Layer Problems, Annals of Mathematics, 43 (1942), pp. 381-407.

[48] Wilkins, J. E., JR., The Converse of a Theorem of Tchaplygin on Differential Equations, Bulletin of the American Mathematical Society, 53 (1947), pp. 126-129.

The RAND Corporation

Santa Monica, California 